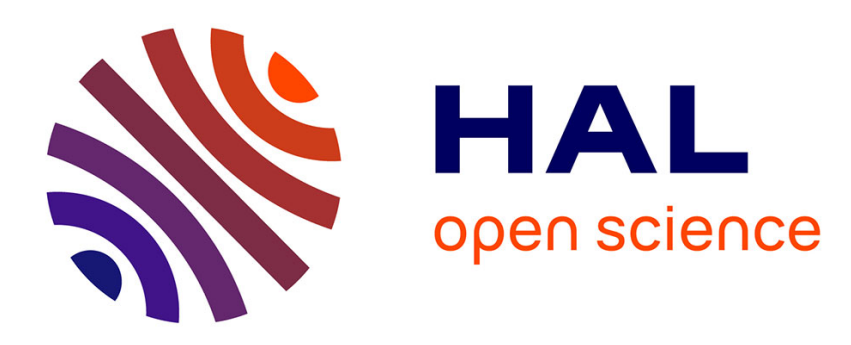

\title{
Global instabilities in spatially developing flows: Non-normality and nonlinearity \\ Jean-Marc Chomaz
}

\section{To cite this version:}

Jean-Marc Chomaz. Global instabilities in spatially developing flows: Non-normality and nonlinearity. Annual Review of Fluid Mechanics, 2005, 37 (1), pp.357-392. 10.1146/annurev.fluid.37.061903.175810 . hal-01024960

HAL Id: hal-01024960

https://hal-polytechnique.archives-ouvertes.fr/hal-01024960

Submitted on 7 Dec 2017

HAL is a multi-disciplinary open access archive for the deposit and dissemination of scientific research documents, whether they are published or not. The documents may come from teaching and research institutions in France or abroad, or from public or private research centers.
L'archive ouverte pluridisciplinaire $\mathbf{H A L}$, est destinée au dépôt et à la diffusion de documents scientifiques de niveau recherche, publiés ou non, émanant des établissements d'enseignement et de recherche français ou étrangers, des laboratoires publics ou privés. 


\title{
Global Instabilities in Spatially Developing FLOWS: Non-Normality and Nonlinearity
}

\author{
Jean-Marc Chomaz \\ Laboratoire d'Hydrodynamique-LadHyX, CNRS-École Polytechnique, 91128 Palaiseau, \\ France; email: chomaz@ladhyx.polytechnique.fr
}

Key Words absolute/convective instabilities, transient growth, fronts, Global modes, passive and active control

- Abstract The objective of this review is to critically assess the different approaches developed in recent years to understand the dynamics of open flows such as mixing layers, jets, wakes, separation bubbles, boundary layers, and so on. These complex flows develop in extended domains in which fluid particles are continuously advected downstream. They behave either as noise amplifiers or as oscillators, both of which exhibit strong nonlinearities (Huerre \& Monkewitz 1990). The local approach is inherently weakly nonparallel and it assumes that the basic flow varies on a long length scale compared to the wavelength of the instability waves. The dynamics of the flow is then considered as a superposition of linear or nonlinear instability waves that, at leading order, behave at each streamwise station as if the flow were homogeneous in the streamwise direction. In the fully global context, the basic flow and the instabilities do not have to be characterized by widely separated length scales, and the dynamics is then viewed as the result of the interactions between Global modes living in the entire physical domain with the streamwise direction as an eigendirection. This second approach is more and more resorted to as a result of increased computational capability. The earlier review of Huerre \& Monkewitz (1990) emphasized how local linear theory can account for the noise amplifier behavior as well as for the onset of a Global mode. The present survey first adopts the opposite point of view by demonstrating how fully global theory accounts for the noise amplifier behavior of open flows. From such a perspective, there is strong emphasis on the very peculiar nonorthogonality of linear Global modes, which in turn allows a novel interpretation of recent numerical simulations and experimental observations. The nonorthogonality of linear Global modes also imposes severe constraints on the extension of linear global theory to the fully nonlinear régime. When the flow is weakly nonparallel, this limitation is so severe that the linear Global mode theory is of little help. It is then much more appropriate to develop a fully nonlinear formulation involving the presence of a front separating the base state region from the bifurcated state region. 


\section{INTRODUCTION}

Mixing layers, wakes, jets, channel flow, Couette flow, boundary layers, and also Rayleigh-Bénard convection with throughflow or Taylor-Couette flow with axial advection, all belong to the open flow category where fluid particles continuously enter and leave the experimental domain. Such configurations develop strong instabilities and eventually exhibit transition to turbulence. The theoretical description of the dynamics should consider the spatial origin of the flow, the perturbations introduced at the inlet (extrinsic noise or forcing), the advection of the perturbation, the spatial evolution of the basic flow (spatial inhomogeneity), and naturally strong nonlinearities. It should describe the intrinsic behavior (self-sustained oscillations or Global modes) as well as the extrinsic behavior (noise-driven perturbations). The analysis is often made difficult by the spatial extent of the flow because different regions may contribute to the dynamics. Two different points of view have been adopted by considering the fate of perturbations either locally, at each streamwise location, or globally, in the whole physical domain. The local point of view, which is legitimate for weakly nonparallel flows, represents at each streamwise station the perturbations as a superposition of instability waves of the associated parallel flow (see Drazin \& Reid 1981 for the stability of parallel flows and Huerre \& Rossi 1998 for nonparallel extensions). The global behavior of the flow depends on the competition between local instability and basic advection, as formalized via the concepts of absolute and convective instability (Bers 1975, Briggs 1964, Sturrock 1958). An open flow may then be globally linearly stable while being locally convectively unstable because perturbations are constantly transported away from the unstable region. When externally forced, such a flow behaves as an amplifier. Conversely, when the flow is absolutely unstable in a finite region, self-sustained resonances may occur as the result of the linear global instability (Chomaz et al. 1988, Huerre \& Monkewitz 1985, Koch 1985, Monkewitz et al. 1993, Pierrehumbert 1984). Figure 1 presents an example of the self-sustained oscillations occurring in the separated boundary layer flow over a double-bump topography as computed by Marquillie \& Ehrenstein (2003).

Huerre \& Monkewitz (1990) reviewed the connection between local theory and global dynamics, under the double restriction of the linear and weakly nonparallel approximations. In the present survey, recent developments that have relaxed these two limitations are reviewed. Global mode theory, still under the weakly nonparallel approximation, was extended to the fully nonlinear régime with the introduction of the front concept (see the reviews by Huerre 2000 and Soward 2001). With the increase of computer capability, the linear or nonlinear analyses of strongly nonparallel flows, referred therein as the fully global problem, become tractable. Efficient stability solvers were thus proposed by Edwards et al. 1998, Schmid \& Henningson 2001, Theofilis et al. 2002, Tuckerman et al. 2000, and references therein.

In this review, I repeatedly emphasize that the linear evolution operator governing Global modes exhibits a peculiar non-normality due to the basic streamwise 


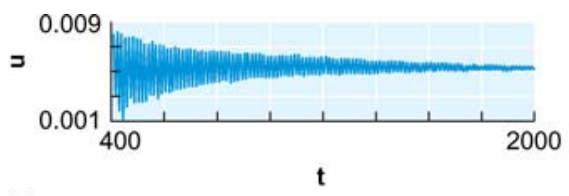

(a)

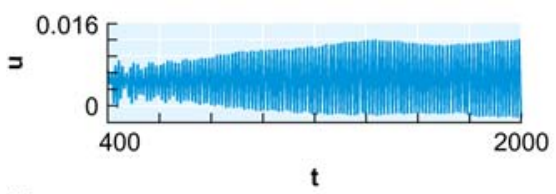

(b)

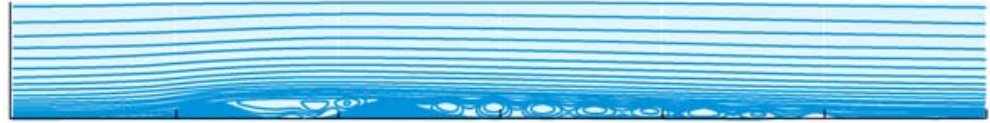

(c)

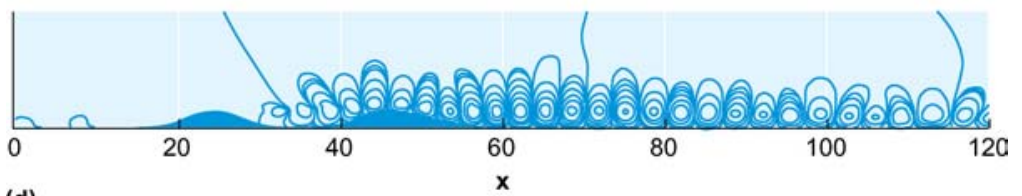

(d)

Figure 1 Global mode in nonparallel flows. Numerical simulation of the separated boundary layer flow over a bottom topography from Marquillie \& Ehrenstein (2003): time history of the streamwise velocity at $x=40, y=1$ for $(a) \operatorname{Re}=850$, (b) $R e=900$. In (a) the flow is globally stable and the perturbation relaxes back to zero with time; in $(b)$ the flow is globally unstable and the perturbation grows and saturates. Saturated Global mode at $R e=900$ : instantaneous streamlines of the total $(c)$ and the perturbation $(d)$ flow field. For $R e=900$ a portion of the flow is absolutely unstable.

advection. To a large extent, this feature dictates the nature of the dynamics, both in the noise-amplifier and self-sustained régime, as argued in section 3 . This property plays an important role in the fully global, strongly nonparallel approach and in the weakly nonparallel formulation. It is claimed to provide an attractive conceptual framework that complements the more classical local instability analyses.

The survey is organized as follows. In section 2.1 basic concepts from the linear theory of Global modes are recalled, as reviewed by Huerre \& Monkewitz (1990). Several standard properties of non-normal operators useful for understanding the fully global dynamics are then summarized in section 2.2. A more comprehensive discussion of these notions is available in the recent book by Schmid \& Henningson (2001). The non-normality of the linear global evolution operator and its implications for the linear and nonlinear dynamics of open flows is the subject of section 3. In particular it is demonstrated that weakly nonlinear theory is adequate 
to describe the dynamics of strongly nonparallel open flows but not of weakly nonparallel flows. For weakly nonparallel flows, section 4 provides a survey of the extensions of absolute and convective instability ideas to the fully nonlinear régime.

\section{FOUNDATIONS}

\subsection{Local Linear Concepts}

This section only intends to give a short summary of the local linear dynamics of open flows. A more exhaustive review can be found in Huerre \& Monkewitz (1990). The successful applications of linear Global mode concepts are numerous and only a few are mentioned here because our goal is to focus on recent fully nonlinear formulations. For simplicity the flow is considered two-dimensional.

2.1.1. LINEAR STABILITY ANALYSIS OF PARALLEL FLOWS Infinitesimal fluctuations around a parallel basic flow given by the streamwise velocity profile $U_{B}(y)(x$ being the streamwise and $y$ the cross-stream coordinates) may be regarded as a combination of elementary instability waves $\mathcal{A} \varphi(y, k, \omega) \exp [i(k x-\omega t)]$ of complex wavenumber $k$, complex frequency $\omega$, amplitude $\mathcal{A}$, and eigenfunction $\varphi(y, k, \omega)$. Depending on the selected representation, $\varphi$ stands either for the velocity and pressure perturbations or for the associated streamfunction. The existence of $\varphi(y, k, \omega)$ constrains $k$ and $\omega$ to satisfy a dispersion relation of the form

$$
D(k, \omega, R)=0,
$$

where $R$ represents the control parameters, for example the Reynolds number. The flow is unstable when temporal modes $\omega(k, R)$ with $k$ real have a positive growth rate $\omega_{i}(k, R)$ for some $k$. This is equivalent to the instability condition $\omega_{i \max }(R)>0$, where $\omega_{i \max }(R)$ is the maximun growth rate over all real $k$.

When a particular frame, the "laboratory" frame, is singled out by forcing at a specific location or by the boundary conditions, the above stability considerations should be complemented with the concepts of absolute and convective instability, which quantify the competition between dispersion induced by the instability and basic advection (Bers 1975, Briggs 1964). For parallel flows, the instability is absolute when the response to a localized initial impulse, Green's function, tends to infinity with time at any fixed location $x$ in the laboratory frame and convective when the impulse response goes to zero in that particular frame but to infinity in at least one Galilean frame. The behavior in the laboratory frame is given by the wave of zero group velocity $d \omega / d k=0$ that corresponds to a saddle point of the dispersion relation located at the absolute wavenumber $k_{0}(R)$ and associated with the absolute frequency $\omega_{0}(R)$ (for a more rigorous derivation and definition that include the pinching condition, see Huerre 2000). If the absolute growth rate $\omega_{0 i}$ is negative, the flow is convectively unstable. If $\omega_{0 i}$ is positive, the instability is absolute. Only when the instability is convective can the response to a localized harmonic forcing be defined because, in the laboratory frame, initial perturbations 
vanish exponentially. When the instability is absolute, initial perturbations grow exponentially everywhere in the laboratory frame and overshadow the response to forcing. The above considerations demonstrate that convectively unstable open flows behave as spatial amplifiers of incoming perturbations. On the contrary, absolutely unstable flows exhibit intrinsic dynamics. As reviewed in Huerre 2000, when the instability is convective, harmonic forcing at the frequency $\omega_{f}$, applied at the location $x=0$ and switched on at time $t=0$, induces exponentially evolving responses that differ upstream and downstream (Figure 2). Upstream of the forcing station the complex wavenumber belongs to the branch $k^{-}$, whereas downstream it belongs to the branch $k^{+}$, both branches being solutions of the dispersion relation (Equation 1), i.e., $D\left(k^{ \pm}, \omega_{f}, R\right)=0$ (see Figure 2). For $x>0$, the downstream response $A_{f}(x, y, t)$ is asymptotic to

$$
A_{f}(x, y, t) \sim \mathcal{A}_{f} \varphi\left(y, k^{+}\left(\omega_{f}\right), \omega_{f}\right) \frac{e^{i\left(k^{+}\left(\omega_{f}\right) x-\omega_{f} t\right)}}{\frac{\partial D}{\partial k}\left(k^{+}\left(\omega_{f}\right), \omega_{f}\right)},
$$

where $\mathcal{A}_{f}$ is the forcing amplitude weighted by a function of the receptivity of the flow to the precise shape of the forcing in the $y$-direction. For $x<0$, a similar formula holds with $k^{+}$replaced by $k^{-}$. When the flow is convectively unstable, all the forcing frequencies with $k_{i}^{+}\left(\omega_{f}\right)<0$ are amplified downstream with a spatial growth rate $-k_{i}^{+}\left(\omega_{f}\right)$.

2.1.2. GLOBAL LINEAR STABILITY ANALYSIS OF WEAKLY NONPARALLEL FLOWS As already stated in the introduction, nonuniformity in $x$, i.e., nonparallelism, means that the basic flow $\mathbf{U}_{B}(x, y)$ now varies not only in the cross-stream direction $y$ but also in the streamwise direction $x$. In this case, the stability analysis has to be performed globally in the whole physical domain by looking for solutions of the form $\phi_{G}(x, y) \exp \left(-i \omega_{G} t\right)$. Here the term "global" refers to the fact that $x$ now has to be considered as an eigendirection. The purpose of this section is to recall essential results, already reviewed by Huerre \& Monkewitz (1990), that link the local instability characteristics at each streamwise $x$-station and the global instability properties, when the flow is weakly nonparallel.

The spatial development of the basic flow may be quantified by the streamwise evolution of a typical local length scale $\delta(x)$ of the velocity profile, for example the local displacement or momentum or vorticity thickness. Then $d \delta / d x$ measures the degree of inhomogeneity of the flow at each station $x$ and its maximum value, $\varepsilon$, the degree of nonparallelism of the entire flow. For $\varepsilon \ll 1$ the basic flow changes over a slow streamwise scale $X=\varepsilon x$ and we may expect that, at each station $X$, the dynamics should be as if the flow were parallel. Within the WKBJ approximation scheme (Bender \& Orzag 1978), perturbations may be decomposed into a slowly varying envelope and a fast varying complex phase $\theta(x, t)$ that should obey the local dispersion relation: $D(k, \omega, R ; X)=0$, with $k=\partial \theta / \partial x$ the local wavenumber, and $\omega=-\partial \theta / \partial t$ the local frequency. For weakly nonparallel flows, which are stable or convectively unstable everywhere, an initially compact perturbation grows while it is traveling in the convectively unstable region but it decays when it reaches 


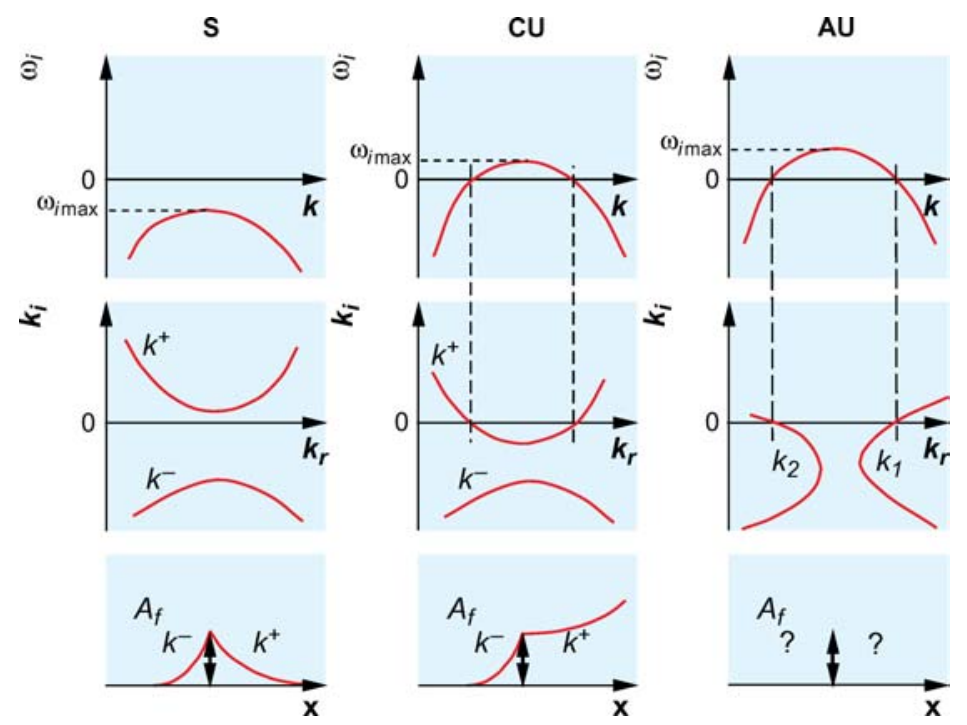

Figure 2 Classical linear stability theory for stable (S), convectively unstable (CU) and absolutely unstable (AU) flow. The first row of sketches illustrates the temporal stability theory with $k$ real (temporal growth rate $\omega_{i}$ versus real wavenumber $k$ ). The second row illustrates the spatial stability theory with $\omega$ real (locus of the complex wavenumber $k$ for varying real frequency $\omega$ ) that describes the response to a harmonic forcing localized at $x=0$. For a stable flow, the response to forcing, schematically shown in the last row, is damped and the $k$-branches that lie above the real $k$-axis propagate to the right of the forcing station. They are therefore labeled with a + sign, whereas $k$-branches that lie below the real $k$-axis propagate to the left and are labeled with a - sign. When a $k^{+}$-branch crosses the real $k$-axis the flow becomes unstable to a particular range of forcing frequencies. For a frequency in this unstable range, the spatial response to forcing is amplified downstream of the forcing station because, by a simple continuation argument, the $k^{+}$-branch keeps propagating downstream. When a $k^{+}$-branch pinches with a $k^{-}$-branch, such an identification by continuity of the direction of propagation is no longer possible, the flow becomes absolutely unstable, and the response to a localized forcing cannot be defined. Any initial transient will then be amplified in situ because the wave with zero group velocity is temporally growing and overwhelms any other signal.

the stable region. As a result, globally stable open flows exhibit large transient growth associated with instability wave propagation downstream, but for a long time and in the absence of external forcing or feedback, they relax to the basic state. They also exhibit large amplification if harmonic forcing at the frequency $\omega_{f}$ is locally applied at the station $x=0$. The downstream response $A_{f}(x, y, t)$ is asymptotic for $x>0$ to 


$$
A_{f}(x, y, t) \sim \mathcal{A}_{f}(X) \varphi\left(y, k^{+}\left(\omega_{f} ; X\right), \omega_{f} ; X\right) e^{i\left(\int_{0}^{X} k^{+}\left(\omega_{f} ; X^{\prime}\right) d X^{\prime}-\omega_{f} t\right)},
$$

where $\mathcal{A}_{f}(X)$ is a slowly evolving amplitude function that Huerre \& Rossi (1998) computed on simple models. All the forcing frequencies with $k_{i}^{+}\left(\omega_{f}, X^{\prime}\right)<0$ at some station $X^{\prime}>0$ experience a finite amplification in some portion of the flow. The gain between $X=0$ and a downstream location $X$ is at leading order $G\left(X, \omega_{f}\right) \sim \exp \left[-\int_{0}^{X} k_{i}^{+}\left(\omega_{f} ; X^{\prime}\right) d X^{\prime}\right]$. For frequencies and downstream locations in a particular range, the gain is larger than unity when the flow is locally unstable and reaches a maximum for a particular forcing frequency $\omega_{f}$ and a particular station $X$. This maximum gain becomes extremely large when the extent of the locally unstable region increases.

By contrast, the existence of a finite region of absolute instability, within the WKBJ approximation scheme $(\varepsilon \ll 1)$, is a necessary condition for global instability to arise through a purely hydrodynamic feedback loop (Chomaz et al. 1991, Monkewitz et al. 1993). For a flow in an infinite domain (Figure $3 b$ ) with a finite absolute instability region, the asymptotic analysis yields a quantitative prediction of the Global mode frequency. Under regularity assumptions for the dispersion relation, the Global mode frequency is given at leading order by $\omega_{G} \sim \omega_{0}\left(X_{S}\right)$, where $X_{S}$ is the complex saddle point such that $\partial \omega_{0} / \partial X\left(X_{S}\right)=0$, or equivalently the double saddle point $\omega\left(k_{S}, X_{S}, R\right)$ with $\partial \omega / \partial k\left(k_{S}, X_{S}, R\right)=0$ and $\partial \omega / \partial X\left(k_{S}, X_{S}, R\right)=0$. At the global threshold, $\omega_{G}$ is real, the absolutely unstable region acts as a wave maker that sheds a downstream $k^{+}\left(\omega_{G} ; X\right)$ wave and an upstream $k^{-}\left(\omega_{G} ; X\right)$ wave. This Global mode structure is similar to the one computed by Soward \& Jones (1983) in Taylor-Couette flow between concentric spheres.

When the flow is semi-infinite and the absolutely unstable region appears at the inlet $X=0$, a Global mode is destabilized when the absolutely unstable region is a few wavelengths large and the Global frequency is then the absolute frequency at the inlet: $\omega_{G}=\omega_{0}(X=0)$ as sketched in Figure $3 a$ (Chomaz et al. 1988, Monkewitz et al. 1993).

The global analysis based on linear absolute instability concepts successfully predicts the occurrence of finite amplitude Global modes in counter-flow mixing layers (Strykowski \& Niccum 1991, Strykowski et al. 1996), wakes with or without suction (Hammond \& Redekopp 1997, Leu \& Ho 2000, Oertel 1990, Woodley \& Peake 1997), hot or helium jets (Kyle \& Sreenivasan 1993, Monkewitz et al. 1990, Sreenivasan et al. 1989, Yu \& Monkewitz 1993), separated boundary layer flows over a double-bump topography (Marquillie \& Ehrenstein 2003; see Figure 1), or even flickering candles (Maxworthy 1999), among many other examples.

In particular, Hammond \& Redekopp (1997), through analytical continuation of the local absolute frequency $\omega_{0}(X)$ for the mean flow extracted from a direct numerical simulation of the wake past a blunt-edged plate, have confirmed that the global frequency is given by the complex saddle point of the local absolute frequency $\omega_{G}=\omega_{0}\left(X_{S}\right)$, as predicted by asymptotic theory. 


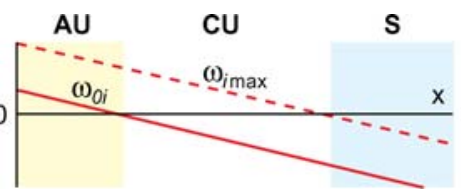

(a)

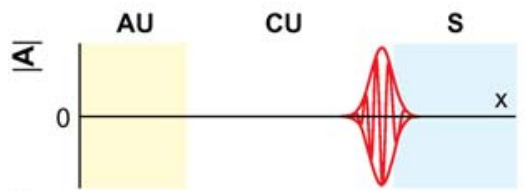

(c)

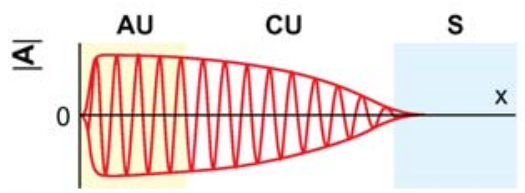

(b)
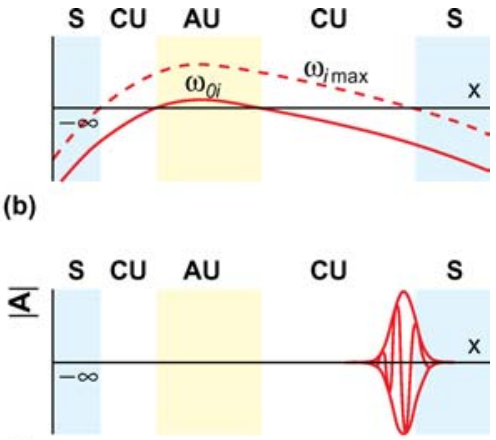

(d)

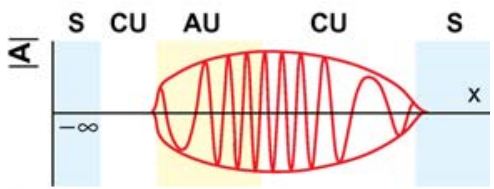

(f)

Figure 3 Linear and nonlinear Global modes in weakly nonparallel flows. ( $a$ ) and $(b)$ illustrate the spatial evolution of the maximum temporal growth rate $\omega_{i \max }$ and absolute growth rate $\omega_{0 i}$. (a) semi-infinite domain with the region of absolute instability (AU) located close to the inlet; $(b)$ infinite domain. The flow is stable when $\omega_{i \max }<0$ (blue), convectively unstable when $\omega_{i \max }>0$ and $\omega_{0 i}<0$ (white), and absolutely unstable when $\omega_{0 i}>0$ (yellow). Linear Global modes: $(c)$ in a semi-infinite domain, $(b)$ in an infinite domain. In both cases the linear Global mode is maximum at the location where $k_{i}^{+}\left(\omega_{G} ; X\right)$ vanishes, e.g., close to the stable region of the flow. In both cases the Global frequency is determined by the absolute frequency in the absolutely unstable domain. In $(c)$ it equals the absolute frequency at the inlet and in $(d)$ the absolute frequency at the complex saddle point such that $\partial \omega_{0} / \partial X\left(X_{S}\right)=0$ (Chomaz et al. 1988, 1991; Monkewitz et al. 1993). The nonlinear Global modes shape presented $(e)$ in a semi-infinite domain and $(f)$ in an infinite domain is in sharp contrast with its linear counterpart. It always consists of a sharp front located at the upstream boundary of the absolute instability domain. In case $(e)$ the Global frequency at the threshold is at leading order the absolute frequency at the inlet $\omega_{G}=\omega_{0}\left(X_{C A}=0\right)$. In case $(f)$ the global frequency is for all parameter values the absolute frequency at the upstream border $X_{A}$ of the absolute instability domain $\omega_{G}=\omega_{0}\left(X_{C A}\right)$ (Harris et al. 2000, Pier et al. 1998), which is in general different from the linear global frequency prediction.

\subsection{Properties of Non-Normal Linear Operators}

To address the fully global problem in section 3 we need to introduce the linear global evolution operator given by the Navier-Stokes equations linearized around the nonparallel basic flow. The spectrum of this operator $\lambda=-i \omega_{G}$ corresponds to the complex frequency of the self-sustained oscillations associated with the 
linear Global modes of spatial distribution $\phi_{G}(x, y)$. In the last decade, it has become abundantly clear that, when the linear evolution operator is non-normal, its spectrum is not sufficient to characterize the dynamics of the flow (Trefethen et al. 1993 and references therein). The present section recalls several useful properties of non-normal operators. The reader should refer to Schmid \& Henningson (2001) for a more comprehensive presentation.

Consider for simplicity the dynamics described by the two degree-of-freedom system:

$$
\frac{d u}{d t}=\mathbf{L} u \text { with } u=\left(\begin{array}{l}
u_{1} \\
u_{2}
\end{array}\right) \text { and } \mathbf{L}=\left(\begin{array}{cc}
-\beta & 0 \\
1 & -\alpha
\end{array}\right),
$$

where $\alpha$ and $\beta$ are complex numbers with positive real parts such that $0<\alpha_{r}<$ $\beta_{r}$ so as to yield a stable system. The eigenmodes of the operator $\mathbf{L}$ are $\phi_{1}=$ $(0,1)^{t}$ associated with the eigenvalue $\lambda_{1}=-\alpha$ and $\phi_{2}=\left(1+|\beta-\alpha|^{2}\right)^{-1 / 2}(\alpha-$ $\beta, 1)^{t}$ associated with the eigenvalue $\lambda_{2}=-\beta$, where the superscipt ${ }^{t}$ denotes the transposed matrix.

This phase space is furthermore endowed with a scalar product $\langle u \mid v\rangle$ defined a priori. This point is crucial because the non-normality concept describes the dynamics of the system (Equation 4) when the magnitude of $u$ is measured through the specific norm $|u|^{2}=\langle u \mid u\rangle$. Then the adjoint operator $\mathbf{L}^{A}$ verifies the equality $\left\langle\mathbf{L}^{A} u \mid v\right\rangle=\langle u \mid \mathbf{L} v\rangle$ for all $u$ and $v$ and the operator $\mathbf{L}$ is non-normal if it does not commute with its adjoint: $\mathbf{L}^{A} \mathbf{L} \neq \mathbf{L} \mathbf{L}^{A}$.

Choosing the Euclidian norm associated with the scalar product $\langle u \mid v\rangle=u_{1}^{*} v_{1}+$ $u_{2}^{*} v_{2}$, where the superscript ${ }^{*}$ denotes the complex conjugate, the adjoint operator $\mathbf{L}^{A}$ is simply the transconjugate matrix $\mathbf{L}^{t *}$ of $\mathbf{L}$. The normed adjoint eigenmodes are $\psi_{1}=\left(1+|\beta-\alpha|^{2}\right)^{-1 / 2}\left(1, \beta^{*}-\alpha^{*}\right)^{t}$ associated with the eigenvalue $\lambda_{1}=-\alpha^{*}$, and $\psi_{2}=(1,0)^{t}$ associated with the eigenvalue $\lambda_{2}=-\beta^{*}$. As shown in Figure 4, the direct and adjoint bases are biorthogonal: $\left\langle\psi_{1} \mid \phi_{2}\right\rangle=\left\langle\psi_{2} \mid \phi_{1}\right\rangle=0$. Because the operator $\mathbf{L}$ is non-normal, the direct and adjoint normed bases do not coincide.

It is now well established that, as a result of non-normality, the perturbation energy may experience transient growth: for the initial condition $u_{0}$, the solution of Equation 4 is $u(t)=\frac{\left\langle\psi_{1} \mid u_{0}\right\rangle}{\left\langle\psi_{1} \mid \phi_{1}\right\rangle} \phi_{1} e^{\lambda_{1} t}+\frac{\left\langle\psi_{2} \mid u_{0}\right\rangle}{\left\langle\psi_{2} \mid \phi_{2}\right\rangle} \phi_{2} e^{\lambda_{2} t}$. When $\left\langle\psi_{1} \mid \phi_{1}\right\rangle$ is small, the magnitude $|u(t)|$ may exhibit large transient growth even though the flow is stable, as seen from the expression of $u(t)$ and Figure 4.

Non-normality may also lead to extreme sensitivity to forcing. Let a steady forcing term $F e^{\lambda_{f} t}$, with $F$ a two-component vector and $\lambda_{f}=-i \omega_{f}$ a prescribed complex frequency be added to the right-hand side of Equation 4 . The response to forcing at large time is then given by $u=U_{f} e^{\lambda_{f} t}$, with $U_{f}=\left(\lambda_{f} \mathbf{I}-\mathbf{L}\right)^{-1} F, \mathbf{I}$ being the identity operator. The operator $(\lambda \mathbf{I}-\mathbf{L})^{-1}$ is known as the resolvent. The border of the region in $\lambda$-space where its norm satisfies $\left\|(\lambda \mathbf{I}-\mathbf{L})^{-1}\right\|>\epsilon$, defines the $\epsilon$-pseudospectrum. Recall that the norm in matrix space is induced by the energy norm in vector space according to the relation $\left\|(\lambda \mathbf{I}-\mathbf{L})^{-1}\right\| \equiv \max _{\|F\|=1} \|(\lambda \mathbf{I}-$ L) ${ }^{-1} F \|$ (Trefethen et al. 1993; Neo 1999, personal communication). When $\lambda_{f}$ is purely imaginary, the resolvent norm coincides with the usual response to a steady 


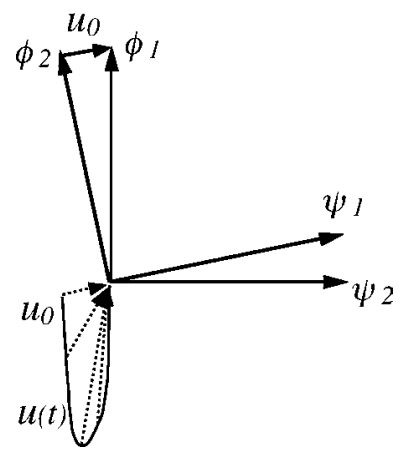

Figure 4 Transient energy growth and non-normality. Sketch of the time evolution of an initial condition $u_{0}=\phi_{2}-\phi_{1}$ (dotted segments) when $\alpha_{r}$ is small and the evolution operator $\mathbf{L}$ given by the model problem (Equation 4 ) is non-normal. Because the scalar product between direct and adjoint eigenvectors $\left\langle\psi_{1} \mid \phi_{1}\right\rangle$ is small, the perturbation issuing from the initial condition $u_{0}$ exhibits large transient growth.

forcing at a real frequency $\omega_{f}$. If the damped operator $\mathbf{L}$ is normal, the largest response corresponds to a gain $G\left(\omega_{f}\right)=\left\|\left(-i \omega_{f} \mathbf{I}-\mathbf{L}\right)^{-1}\right\| \sim 1 / \alpha_{r}$, because $-\alpha_{r}$ is the real part of the eigenvalue that is closest to the imaginary axis, as expected for a damped oscillator. If $\mathbf{L}$ is non-normal, the response to forcing may give rise to a much larger gain given by $1 / \epsilon_{0}, \epsilon_{0}$ being the smallest value of $\epsilon$ for which the $\epsilon$-pseudospectrum crosses the imaginary $\lambda$-axis.

Finally, the non-normality of the operator $\mathbf{L}$ induces a large sensitivity of the spectrum to perturbations. If, in Equation $4, \mathbf{L}$ is replaced by $\mathbf{L}+\epsilon \Delta \mathbf{L}, \Delta \mathbf{L}$ being a perturbation operator of a prescribed norm $\|\Delta \mathbf{L}\|=1$ and the operator $\mathbf{L}$ being assumed of norm close to unity, then the eigenvalues of the perturbed problem will move inside the previously defined $\epsilon$-pseudospectrum (Schmid \& Henningson 2001, Trefethen et al. 1993). When $\mathbf{L}$ is non-normal the $\epsilon$-pseudospectrum contour may be at a distance much larger than $\epsilon$ from the spectrum and small perturbations of the evolution operator $\mathbf{L}$ may induce large modification of the spectrum.

If only the sensitivity of the individual eigenvalue $\lambda_{1}$ to perturbations of the operator $\mathbf{L}$ is of interest, then the computation of the $\epsilon$-pseudospectrum is not necessary. A simple expansion procedure, $\phi_{1}^{\prime} \sim \phi_{1}+\epsilon \delta \phi_{1}$ and $\lambda_{1}^{\prime} \sim \lambda_{1}+\epsilon \delta \lambda_{1}$, gives, when the relation $(\mathbf{L}+\epsilon \Delta \mathbf{L}) \phi_{1}^{\prime}=\lambda_{1}^{\prime} \phi_{1}^{\prime}$ is expanded to order $\epsilon$ :

$$
\delta \lambda_{1}=\frac{\left\langle\psi_{1} \mid \Delta \mathbf{L} \phi_{1}\right\rangle}{\left\langle\psi_{1} \mid \phi_{1}\right\rangle} .
$$

The eigenvalue perturbation $\delta \lambda_{1}$ may become extremely large when non-normality is large, i.e., $\left\langle\psi_{1} \mid \phi_{1}\right\rangle$ is small, because it is possible to choose $\Delta \mathbf{L}$ with $\|\Delta \mathbf{L}\|=1$ such that $\left\langle\psi_{1} \mid \Delta \mathbf{L} \phi_{1}\right\rangle \geq 1$, the equality being guaranteed by the particular choice of $\Delta \mathbf{L}$ defined by $\Delta \mathbf{L} \phi_{1}=\psi_{1}$ and $\Delta \mathbf{L} \psi_{2}=\phi_{2}$. 


\section{FULLY NONLINEAR GLOBAL MODES AND NON-NORMALITY}

Bifurcation theory, which follows the series of transitions affecting a flow when a control parameter $R$, for example the Reynolds number, is increased, provides a way to analyze the global dynamics of open flows. As a first step, it requires computing the stability of the steady basic flow that issues continuously from the rest state. Mamun \& Tuckerman (1995) computed the global mode bifurcation for the spherical Couette flow. For the cylinder wake, the primary and secondary instabilities were studied by Jackson (1987), Zebib (1987), Noack \& Eckelmann (1994), and Barkley \& Henderson (1996), among others. Theofilis (2003) reviewed aeronautical applications of global stability computation. But the effort has mainly been focused on deriving the linear spectrum and the corresponding central manifold theory near threshold (Hopf bifurcation and associated Landau equation), and less attention has been paid to the dynamics arising from the non-normality of the evolution operator, due to the open nature of the flow (Aiken et al. 2003, Chomaz et al. 1990, Cossu \& Chomaz 1997, Giannetti \& Luchini 2003, Lauga \& Bewley 2002, Le Dizès et al. 1993, Moore et al. 2002, Reddy \& Trefethen 1994, Schmid \& Henningson 2002). If perturbations around a basic flow are expressed in terms of the velocity field $\mathbf{u}$, and the perturbation magnitude measured by the energy norm $\int \mathbf{u}^{2} d \mathbf{x}$, then the basic flow advection term $\mathbf{U}_{B} \nabla \mathbf{u}$ in the linearized evolution operator $L$ gives, after suitable integration by parts (Schmid \& Henningson 2001), the term $-\mathbf{U}_{B} \nabla \mathbf{u}^{\prime}$ in the adjoint operator. This basic flow advection term effectively corresponds to upstream transport for the adjoint perturbations and it is mainly responsible for the non-normality of the linear evolution operator. We demonstrate below how this non-normality is specific to open flows and how it affects the bifurcation scenario. This non-normality differs from that involved in the bypass transition of Couette or Poiseuille flows, which originate in the tilting of the basic flow vorticity by the perturbation (Butler \& Farrell 1992, Trefethen et al. 1993).

\subsection{Global Bifurcation on a Simple Model}

Consider first the simple one-dimensional amplitude equation of Chomaz et al. (1990):

$$
\frac{\partial A}{\partial t}+L\left(\frac{\partial}{\partial x}, x, R\right) A+c(x, R)|A|^{2} A=f(x, t),
$$

where $R$ represents the control parameter, $L$ the linear differential operator, specified later, such that $L\left(\frac{\partial}{\partial x}, x, R\right) A=0$ for $A=0, A(x, t)$ being a complex scalar field, and $c(x, R)$ the complex weight of the nonlinearity. Many other forms for the nonlinear term may easily be introduced and the one chosen presently is in the spirit of normal form theory (Guckenheimer \& Holmes 1983). The term $f(x, t)$ represents the external forcing. In the absence of forcing the base state (also called basic flow for convenience) $A=0$ is the solution of Equation 6. Assume that 
the domain is infinite and that $A(x, t)$ vanishes exponentially at infinity (finite or semi-infinite domains may also be considered) (Chomaz \& Couairon 1999). The linear equation associated with Equation 6 admits linear Global modes of the form $A_{G}(x, t)=\phi_{G}(x) \exp \left(-i \omega_{G} t\right)$, and only the most unstable mode is of interest to define the stability of the basic flow. The complex frequency $\omega_{G}$ and the eigenmode $\phi_{G}(x)$ depend on $R$ and a critical value $R_{c}$ exists such that the base state $A=0$ is globally stable for $R<R_{c}$. At $R=R_{c}$, the system is neutral and the global frequency $\omega_{c}=\omega_{G}\left(R_{c}\right)$ is real. In this case, the amplitude of the leading mode evolves slowly with respect to the time scale $\omega_{c}^{-1}$ assumed finite. A standard multiple-scale analysis (Nayfeh 1973) is performed by introducing the small parameter $\eta$, which measures the departure from criticality so that $R=R_{c}+\Delta_{R} \eta^{2}, f(x, t)=\eta^{2} F \delta\left(x-x_{f}\right) \exp \left(-i \omega_{f} t\right), \omega_{f}=\omega_{c}+\eta^{2} \Omega$. A slow time scale $T=\eta^{2} t$ is introduced and perturbations are expanded according to $A(x, t)=\sum_{n=1}^{\infty} \eta^{n} A_{n}(x, t, T)$. Assuming the operator $L$ is differentiable with respect to $R$, the operator differentiated with respect to $R$ is noted as $L_{R}$ and, allowing the operator $L$ to be perturbed at order $\eta^{2}$ by a given operator $\Delta L$, we may write

$$
L\left(\frac{\partial}{\partial x}, x, R\right)=L\left(\frac{\partial}{\partial x}, x, R_{c}\right)+\eta^{2} \Delta_{R} L_{R}\left(\frac{\partial}{\partial x}, x, R_{c}\right)+\eta^{2} \Delta L+O\left(\eta^{4}\right) .
$$

Thus, the leading-order equation corresponds to the linear eigenvalue problem at threshold

$$
\frac{\partial A_{1}}{\partial t}+L\left(\frac{\partial}{\partial x}, x, R_{c}\right) A_{1}=0
$$

and it admits the solution $A_{1}(x, t, T)=\mathcal{A}(T) \phi_{G}(x) \exp \left(-i \omega_{c} t\right)$, i.e., the neutral Global mode of amplitude $\mathcal{A}(T)$. Here the function $\phi_{G}(x)$ stands for the eigenfunction at threshold $R_{c}$ and is assumed of norm unity $\left(\int\left|\phi_{G}(x)\right|^{2} d x=1\right)$. The next-order term $A_{2}$ satisfies the same homogeneous equation as Equation 8 and provides no essential information or constraint concerning $\mathcal{A}(T)$. A compatibility condition for the elimination of secular terms at third order leads to the perturbed Landau equation for the Global mode amplitude

$$
\begin{aligned}
\frac{d \mathcal{A}}{d T}= & \Delta_{R} \frac{\left\langle\psi_{G} \mid L_{R} \phi_{G}\right\rangle}{\left\langle\psi_{G} \mid \phi_{G}\right\rangle} \mathcal{A}-\frac{\left\langle\left.\psi_{G}|c(x, R)| \phi_{G}\right|^{2} \phi_{G}\right\rangle}{\left\langle\psi_{G} \mid \phi_{G}\right\rangle}|\mathcal{A}|^{2} \mathcal{A} \\
& +\frac{\left\langle\psi_{G} \mid \Delta L \phi_{G}\right\rangle}{\left\langle\psi_{G} \mid \phi_{G}\right\rangle} \mathcal{A}-F \frac{\psi_{G}^{*}\left(x_{f}\right)}{\left\langle\psi_{G} \mid \phi_{G}\right\rangle} \exp (-i \Omega T) .
\end{aligned}
$$

The quantity $\langle f \mid g\rangle$ denotes the scalar product $\int f^{*} g d x$ and $\psi_{G}(x)$ is the adjoint Global mode of norm unity, solution of the equation $\left[i \omega_{c}^{*}+L^{A}\left(\frac{\partial}{\partial x}, x, R_{c}\right)\right] \psi_{G}=$ 0 , where $L^{A}$ is the adjoint operator of $L$.

Equation 9 is the master equation that is needed to understand the dynamics of open flows from a global point of view. The first term on the right-hand side represents the variation of the Global mode growth rate with the control parameter and it may be rewritten as $-i \Delta_{R} \partial \omega_{G}\left(R_{c}\right) / \partial R$, the second term describes the global 
cubic nonlinear effect, the third term the sensitivity of the eigenvalue $\omega_{G}$ to small changes $\eta^{2} \Delta L$ in the linear evolution operator $L$, and the last term the receptivity of the flow to localized forcing.

Figure $5 a, b$ illustrates the eigenfunction of the direct operator $L$ and its adjoint operator $L^{A}$ for the Ginzburg-Landau equation, in which case $L$ and $L^{A}$ reduce to

$$
L \equiv U \frac{\partial}{\partial x}-\mu(x)-\gamma \frac{\partial^{2}}{\partial x^{2}}, \quad L^{A} \equiv-U \frac{\partial}{\partial x}-\mu^{*}(x)-\gamma^{*} \frac{\partial^{2}}{\partial x^{2}},
$$

with $U$ the basic advection velocity, and $\gamma=1+i c_{d}$ the diffusion and dispersion coefficients $\left(U, \mu, c_{d}\right.$ real). For instance, the amplification parameter $\mu(x)$ exhibits quadratic variations in $x$ of the form $\mu(x)=\mu_{0}+\mu_{2} x^{2} / 2$, with $\mu_{2}<0$. As noticed previously, the advection acts in opposite directions for the direct and adjoint operators. The eigenvalue, the direct eigenfunction (i.e., the direct Global mode) and the adjoint eigenfunction (i.e., the adjoint Global mode) are

$$
\begin{aligned}
-i \omega_{G} & =\mu_{0}-\frac{U^{2}}{4 \gamma}-(2 n+1)\left|\frac{\mu_{2} \gamma}{2}\right|^{1 / 2} e^{i\left(\arg \left(-\mu_{2}\right)+\arg (\gamma)\right) / 2}, \\
\phi_{G_{n}}(x) & =\zeta_{n} H_{n}(\chi x) e^{U x / 2 \gamma-\chi^{2} x^{2} / 2} \\
\psi_{G}(x) & =\xi_{n} \phi_{G n}^{*}(x) e^{-U x / \gamma^{*}} \\
\left\langle\psi_{G_{1}} \mid \phi_{G_{1}}\right\rangle & =\zeta_{1} \xi_{1}\left(\frac{2 \gamma \pi^{2}}{\mu_{2}}\right)^{1 / 4} e^{-i\left(\arg \left(-\mu_{2}\right)-\arg (\gamma)\right) / 4},
\end{aligned}
$$

where $\zeta_{n}$ and $\xi_{n}$ are suitable normalization factors such that $\left\|\phi_{G_{n}}\right\|=1$ and $\left\|\psi_{G_{n}}\right\|=1, \chi=\left|\mu_{2} / 2 \gamma\right|^{1 / 4} \exp \left[i\left(\arg \left(-\mu_{2}\right)+\arg (\gamma)\right) / 4\right], n=0,1,2, \ldots$ and $H_{n}$ is the $\mathrm{n}$-th order Hermite polynomial. The most unstable Global mode corresponds to $n=1$. The eigenfunction basis is orthogonal, i.e., the operator $L$ is normal, only when $U=0$. When $U$ is nonzero, $L$ is non-normal in a specific way because the larger the advection $U$ and the weaker the nonparallelism of the flow, measured here by $\mu_{2}$, the more separated in space the direct Global mode $\psi_{G}$ and the adjoint Global mode $\phi_{G}$ become (Figure 5a,b). This non-normality by spatial separation between direct and adjoint modes differs from the non-normality that comes from the degeneracy of two or more direct modes, as in Poiseuille or Couette flows. In the latter cases, adjoint and direct modes have the same support (Butler \& Farrell 1992, Trefethen 1997). The system is globally unstable if $\mu_{0}>\mu_{c}$ with

$$
\mu_{c} \equiv \frac{U^{2}}{4|\gamma|^{2}}+\left|\frac{\mu_{2} \gamma}{2}\right|^{1 / 2} \cos \left[\left(\arg \left(-\mu_{2}\right)+\arg (\gamma)\right) / 2\right] .
$$

Close to this threshold value $\mu_{c}$, the Landau equation (Equation 9) applies, and the following section demonstrates how the specific non-normality of the linear evolution operator influences both the linear and nonlinear dynamics.

3.1.1. RESPONSE TO FORCING, AMPLIFIER BEHAVIOR, AND TRANSIENT GROWTH The last term of Equation 9 quantifies the response to localized harmonic forcing, 
$\Omega$ real, also known as open-loop control. Assuming that the flow is globally stable, i.e., that $\Delta_{R}$ is negative, and neglecting the nonlinear term, the amplitude of the global response becomes

$$
\mathcal{A}=F \frac{\psi_{G}^{*}\left(x_{f}\right)}{\left\langle\psi_{G} \mid \phi_{G}\right\rangle} \frac{i\left(\Delta_{R} \frac{\partial \omega_{G}^{*}}{\partial R}\left(R_{c}\right)-\Omega\right)}{\left(\Delta_{R} \frac{\partial \omega_{G i}}{\partial R}\left(R_{c}\right)\right)^{2}+\left(\Delta_{R} \frac{\partial \omega_{G r}}{\partial R}\left(R_{c}\right)-\Omega\right)^{2}} \exp (-i \Omega T) .
$$

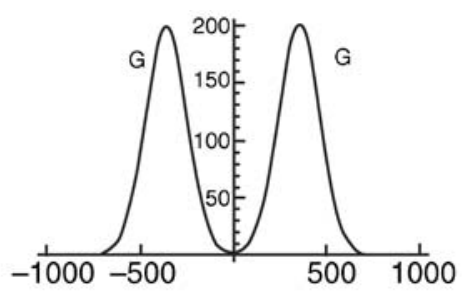

(a)

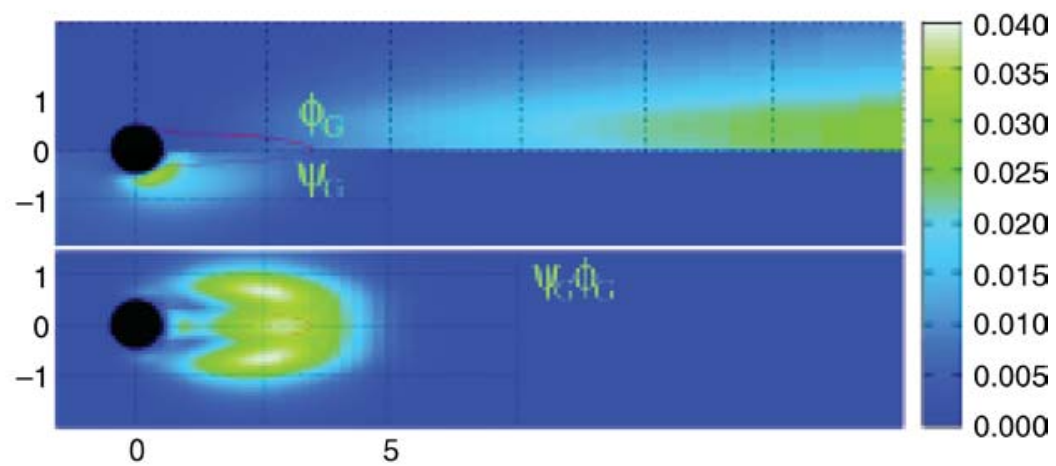

(c)

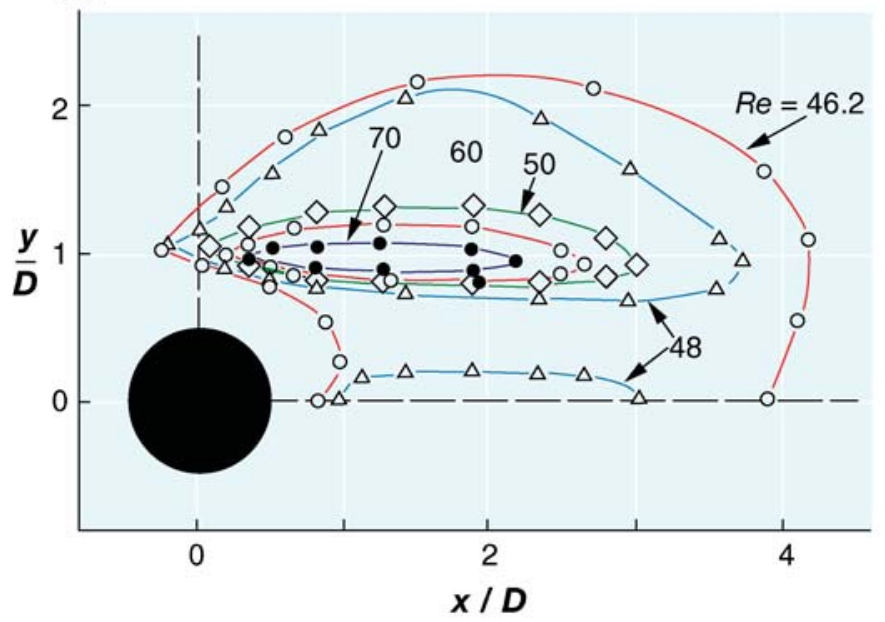

(d)

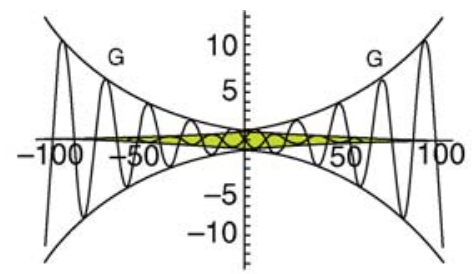

(b)

0.040
0.035
0.030
0.025
0.020
0.015
0.010
0.005
0.000

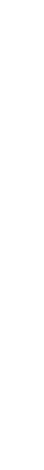


Two different ratios dictate the amplitude of the response. The factor $i\left(\Delta_{R} \frac{\partial \omega_{G}^{*}}{\partial R}\left(R_{c}\right)-\Omega\right) /\left[\left(\Delta_{R} \frac{\partial \omega_{G i}\left(R_{c}\right)}{\partial R}\right)^{2}+\left(\Delta_{R} \frac{\partial \omega_{G r}}{\partial R}\left(R_{c}\right)-\Omega\right)^{2}\right]$ characterizes the effect of the detuning. It is similar to the one encountered for the usual response of a damped linear oscillator and it decreases when the detuning $\left(\Delta_{R} \frac{\partial \omega_{G r}}{\partial R}\left(R_{c}\right)-\Omega\right)$ increases. The factor $\psi_{G}^{*}\left(x_{f}\right) /\left\langle\psi_{G} \mid \phi_{G}\right\rangle$ encompasses the effect of the forcing location and of the non-normality of the operator. The adjoint Global mode measures the receptivity to forcing via the term $\psi_{G}^{*}\left(x_{f}\right)$ : the most efficient forcing station $x_{f}$ is located upstream, at the maximum of $\psi_{G}$. Furthermore, the forcing response is inversely proportional to $\left\langle\psi_{G} \mid \phi_{G}\right\rangle$. Therefore, it may become extremely large when the non-normality increases. From the global point of view the amplifier behavior of globally stable open flows is a direct consequence of the non-normality of the global linear evolution operator. It may be easily quantified because solving the adjoint problem requires the same computer power and may use the same algorithm as the direct stability problem.

According to the linear theory of non-normal operators, the harmonic amplifier behavior is also associated with transient growth of initial perturbations. The optimal initial perturbation for which the energy of the response is the largest after a time $t$ may be computed by using the direct and the adjoint operator. When the non-normality is large, the globally stable flow sustains, close to the global instability threshold, extremely large transient growth of the initial perturbation energy, as demonstrated by Cossu \& Chomaz (1997) on the basis of the results of Hunt \& Crighton (1991) for the Ginzburg-Landau equation (Equations 6 and 10). Figure $6 a$ illustrates the gain in energy over all initial perturbations and time intervals. Below the global instability threshold $\mu_{c}\left(\mu_{2}\right)$, transient growth may be very large when $\mu_{2}$ is small, i.e., when the flow is weakly nonparallel. Therefore, transient growth may be interpreted in two different ways: From the local point of view it is associated with convective instability, whereas from a global point of view, it is the result of the non-normality of the Global evolution operator.

Figure 5 Direct and adjoint Global modes: $(a, b)$ for the Ginzburg-Landau equation with varying coefficients; $(c)$ for the cylinder wake at $R e=50$ (adapted from Giannetti \& Luchini 2003); (d) regions where the addition of a small control cylinder effectively restabilizes the cylinder wake at different Reynolds numbers $R e$ (adapted from Strykowski \& Sreenivasan 1990). (a) Envelope of $\phi_{G}(x)$ and $\psi_{G}(x) ;(b)$ zoom of $(a)$ in the overlapping region (orange) showing the envelope and the real part of the direct and adjoint modes. (c) top image, upper half: local energy of the direct Global mode $\left|\phi_{G}(x, y)\right|^{2}$; top image, lower half: local energy of the adjoint Global mode $\left|\psi_{G}(x, y)\right|^{2}$; lower image: local scalar product of the adjoint and direct Global mode velocities $\left|\psi_{G}(x, y)^{*} \phi_{G}(x, y)\right|$. The color bar corresponds to the field $\left|\psi_{G}(x, y)^{*} \phi_{G}(x, y)\right|$ in the lower image. The correlation is striking between the theoretically most sensitive region to flow modification quantified by $\left|\psi_{G}(x, y)^{*} \phi_{G}(x, y)\right|$ [lower image of $(c)$ ] and the experimentally determined region for the controllability of vortex shedding by the addition of a small cylinder $(d)$. 


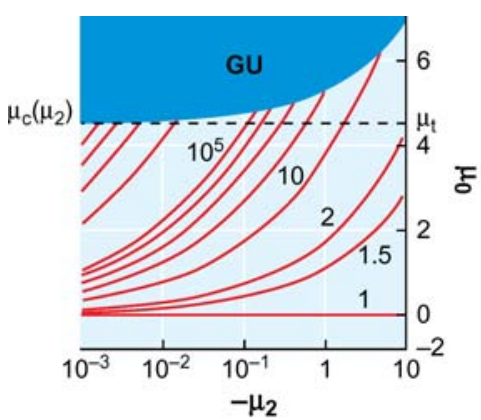

(a)

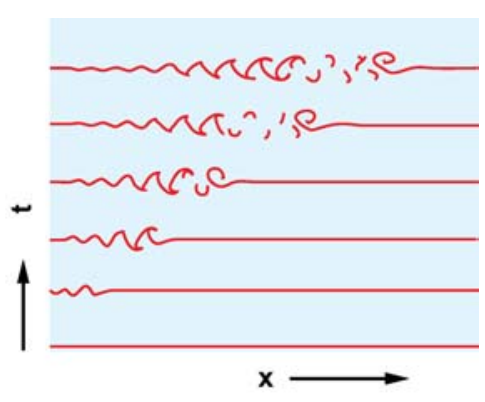

(b)

Figure 6 Transient growth in non-parallel flows: (a) For the Ginzburg Landau Equation 6 (adapted from Cossu \& Chomaz 1997), contour levels of maximum growth $M$ ( $M$ is the gain in perturbation energy maximized over all initial perturbations and over time) as a function of $\mu_{2}$ and $\mu_{0}$ with $\gamma=1-i, U=6 . M=\infty$ in the globally unstable (GU) region. The contour levels are $1.5,2,10,10^{2}, 10^{3}, 10^{4}, 10^{5}, 10^{10}, 10^{15}$, $10^{25}, \ldots .(b)$ Transient growth observed in the wake of a cylinder below the global instability threshold at $R e=35$ (adapted from Le Gal \& Croquette 2000). Waterfall presentation of the streakline generated by a passive tracer visualizing the impulse response in a cylinder wake versus time.

3.1.2. SENSITIVITY OF THE GLOBAL SPECTRUM, EFFECT OF FEEDBACK AND CLOSEDLOOP CONTROL As argued in the foundation section 2.2, the spectrum of nonnormal operators may not be a robust entity because small perturbations of the operator may displace the eigenvalues in a significant manner. This sensitivity of the global spectrum to operator perturbations is represented by the third term on the right-hand side of Equation 9. Changes in the most unstable eigenvalue $\omega$ induced by the operator perturbation $\eta^{2} \Delta L$ are given by:

$$
\omega-\omega_{G}(R)=-i \eta^{2} \frac{\left\langle\psi_{G} \mid \Delta L \phi_{G}\right\rangle}{\left\langle\psi_{G} \mid \phi_{G}\right\rangle} .
$$

Let us first consider local perturbations of the operator $L$, i.e., modifications such that $\Delta L \phi_{G}$ at location $x$ depends only on the value of $\phi_{G}$ and its derivative at $x$. In the present toy model, these perturbations are associated with basic flow modifications such as local variations in the advection velocity $U$, in the amplification parameter $\mu(x)$, or in the diffusion coefficient $\gamma$. For instance, if the local amplification parameter $\mu(x)$ is locally modified by an amount $\eta^{2} \delta \mu(x)$, then contributions to $\left\langle\psi_{G} \mid \Delta L \phi_{G}\right\rangle=\int \psi_{G}^{*}(x) \delta \mu(x) \phi_{G}(x) d x$ will primarily come from domains where $\psi_{G}^{*}(x) \phi_{G}(x)$ is nonzero. Thus, local perturbations will have a large impact if they occur in the overlapping region between the adjoint and direct Global modes. Conversely, if they occur in the region where one of these two modes is vanishing, the induced modification of the spectrum will be very small. 
In physical terms, this means that the region that is crucial in determining the Global mode frequency, the wave maker, is the region of space where the adjoint and the direct Global mode overlap because the spectrum is basically insensitive to modifications of the basic flow outside this region. Therefore, the region of space where the eigenfunction is large does not play a special role in determining the spectrum of a stability equation. This is extremely good news for numerical simulations because it is sufficient to take a numerical domain that includes the wave maker region to properly capture the Global mode dynamics. The objective identification of the wave maker region valid for any nonparallelism of the flow is due to Luchini (2003, personal communication) and Giannetti \& Luchini (2003).

If nonlocal perturbations are allowed, $\Delta L \phi_{G}$ at station $x$ is now a function of $\phi_{G}$ at all stations $x^{\prime}$. For example, $g$ being an arbitrary function, $\Delta L g\left(x^{\prime}\right)=$ $C \delta\left(x^{\prime}-x_{1}\right) g\left(x_{2}\right)$ corresponds to a perturbation of the original problem by a weak feedback loop of order $\eta^{2}$ acting at the actuator station $x_{1}$ and proportional to the response at the sensor station $x_{2}, C$ being the proportionality constant of order unity. The scalar product $\left\langle\psi_{G} \mid \Delta L \phi_{G}\right\rangle=C \psi_{G}^{*}\left(x_{1}\right) \phi_{G}\left(x_{2}\right)$ may now become much larger than $\left\langle\psi_{G} \mid \phi_{G}\right\rangle$ when $x_{1}$ is chosen close to the maximum of the adjoint Global mode where the flow is most receptive and $x_{2}$ close to the maximum of the direct Global mode where the response of the flow is the largest. Such a feedback loop therefore has a huge impact on the global eigenvalues.

Closed-loop control may be viewed as a special case of operator perturbation $\eta^{2} \Delta L$ specifically designed to restabilize the linear evolution operator $L$. For example, the proportional feedback control of the Global mode for the complex Ginzburg-Landau equation with varying coefficients similar to Equation 10 implemented by Roussopoulos \& Monkewitz (1996) corresponds to the feedback loop discussed above with the actuator location at $x_{1}=0$ and the sensor location at $x_{2}=1.5$. In this case, $\omega-\omega_{G}(R)=-i \eta^{2} C \psi_{G}^{*}\left(x_{1}\right) \phi_{G}\left(x_{2}\right)\left\langle\psi_{G} \mid \phi_{G}\right\rangle^{-1}$ and for any given $x_{1}$ and $x_{2}$, it is possible to restabilize the flow near the threshold by choosing the amplitude and phase of the complex gain $C$ in a particular range. More efficient ways to stabilize the flow far from threshold, when several Global modes are unstable, were explored by Lauga \& Bewley (2004) for a single sensor associated with a compensator to reconstruct the flow, and by Lauga \& Bewley (2002) for full-information control with a single actuator. In the present framework, the latter situation corresponds to choosing a perturbation operator where the actuator is localized at $x_{1}$ with an intensity that now depends on the response $A(x, t)$ at all stations in the physical domain. Therefore, it is given by $\Delta L g\left(x^{\prime}\right)=\delta\left(x^{\prime}-x_{1}\right) F(g)$, where $F$ is a scalar function acting, in function space, on $g$. Close to the threshold, the eigenvalue modification $\omega-\omega_{G}(R)=-i \eta^{2} \psi_{G}^{*}\left(x_{1}\right) F\left(\phi_{G}\right)\left\langle\psi_{G} \mid \phi_{G}\right\rangle^{-1}$, reveals that many functionals $F(g)$ may be designed to restabilize the flow. In a more general setting, optimal control theory was shown by Lauga \& Bewley (2002) to yield a functional $F(g)$ capable of restabilizing up to 13 Global modes far above the threshold. For each mode, the influence of the control is limited by the adjoint Global mode $\psi_{G_{n}}\left(x_{1}\right)$, which must be large enough for the actuator to be effective. Because adjoint Global modes become widely separated, this requirement can 
only be met for a finite number of Global modes, which is a function of numerical accuracy: Round-off errors impose limitations on controllability.

3.1.3. EFFECT OF NON-NORMALITY ON THE NATURE OF THE BIFURCATION The Landau equation (Equation 9) describes the weakly nonlinear Global mode dynamics close to the threshold. In the absence of forcing and operator perturbation, it may be rewritten as

$$
\frac{d \mathcal{A}}{d T}=-i \Delta_{R} \frac{\partial \omega_{G}}{\partial R}\left(R_{c}\right) \mathcal{A}-l|\mathcal{A}|^{2} \mathcal{A},
$$

where $l=\left\langle\left.\psi_{G}|c(x, R)| \phi_{G}\right|^{2} \phi_{G}\right\rangle /\left\langle\psi_{G} \mid \phi_{G}\right\rangle$ is the Landau constant computed in Chomaz et al. (1990) when $c$ is independent of $x$. When the non-normality of the evolution operator is moderate, $l$ is of order unity and positive when $c$ is positive (supercritical bifurcation). But when $\mu_{2}$ diminishes, the adjoint and the direct Global modes separate in the streamwise direction, the non-normality increases and $l$ vanishes exponentially with decreasing $\mu_{2}$. In this case, the nonlinear term $\left|\phi_{G}\right|^{2} \phi_{G}$ is unable to saturate the Global mode because nonlinearities are intense where the Global mode is intense, i.e., downstream, whereas the flow is more receptive upstream where the adjoint mode is large. The bifurcation is then very steep, with a saturation amplitude that reaches unity for a super-criticality parameter $\Delta_{R}$ exponentially small with respect to $\mu_{2}$. In this weakly nonparallel case, the Global mode becomes fully nonlinear even for an exponentially small departure from criticality $\Delta_{R}$. The strongly nonlinear Global mode, which prevails above threshold for weakly nonparallel flows, is described in section 4. By contrast, when nonparallelism is large ( $\mu_{2}$ not small), non-normality is moderate and the weakly nonlinear approximation presented here remains valid. The usual Landau equation holds.

\subsection{Global Bifurcation of Real Open Flows}

The specific non-normality due to the basic flow advection is also present in real open flows. Giannetti \& Luchini (2003) developed these ideas for the twodimensional (2D) cylinder wake by extending the earlier work of Hill (1992). Similar considerations are emerging in the geophysical fluid dynamics community: For example, non-normality due to the effect of basic flow advection has been considered by Moore et al. (2002) and Aiken et al. (2003). The previous discussion based on model equations directly transposes to the Navier-Stokes equations and, in the following, this analogy is drawn and new interpretations of specific open flow behaviors are proposed.

Figure 5c, adapted from Giannetti \& Luchini (2003), presents the energy distribution of the direct and adjoint Global modes in the cylinder wake for the linear evolution operator $L$ obtained by linearizing the Navier-Stokes equations around the unstable steady wake solution at $R e=50$. For convenience, the same notation $\phi_{G}, \psi_{G}$ for the direct and adjoint modes is kept. They now symbolize velocity and pressure fields in a $2 \mathrm{D}$ domain. 
Close to the threshold, a slow time-scale analysis may be performed by using an expansion procedure similar to the one implemented on the toy model (Equation 6) in section 3.1. Note that because the flow is not assumed weakly nonparallel, there is no need to introduce a slow space variable. At first order, the linear Global mode is recovered with an arbitrary amplitude that depends on the slow time scale $T, A_{1}(x, y, t, T)=\mathcal{A}(T) \phi_{G}(x, y) \exp \left(-i \omega_{c} t\right)+c c$, where $c c$ stands for the complex conjugate and $A_{1}$ now for the perturbation velocity and pressure fields. The second-order problem is more complex because the advection of the Global mode by the Global mode generally gives two forcing terms at zero frequency and at $2 \omega_{c}$, respectively, which are intense downstream where the Global mode is large. For simplicity, let us assume that only the zero frequency term is present. It induces a mean flow distortion computed by inverting the evolution operator $L$ of the form

$$
A_{2}(x, y, t, T)=|\mathcal{A}|^{2} \phi_{B}(x, y) .
$$

At third order, the compatibility condition necessary to avoid secular terms leads to the Landau equation (Equation 9) except that the coefficient of the nonlinear term $|\mathcal{A}|^{2} \mathcal{A}$ is now $\left\langle\psi_{G} \mid N\left(\phi_{B} \phi_{G}\right)\right\rangle /\left\langle\psi_{G} \mid \phi_{G}\right\rangle$, where the nonlinear operator $N$ stands for all third-order terms coming from the interaction between the Global mode and the mean flow distortion (see Fauve 1998 for a general discussion of amplitude equations in fluid mechanics). This term quantifies the changes in the eigenvalue of the linear operator due to the nonlinear mean flow distortion.

3.2.1. RESPONSE TO FORCING, AMPLIFIER BEHAVIOR, AND TRANSIENT GROWTH When the steady wake flow is stable, the response to a local forcing obeys Equation 13. It is determined by the magnitude of the adjoint Global mode at the forcing location $\left|\psi_{G}\left(x_{f}, y_{f}\right)\right|$ and it is most efficient at the maximum of $\left|\psi_{G}\left(x_{f}, y_{f}\right)\right|$ over all $x_{f}$ and $y_{f}$. For the cylinder wake, Figure $5 c$ demonstrates that the region most receptive to velocity perturbations is located immediately downsream of the cylinder (Giannetti \& Luchini 2003). The maximum amplification is still inversely proportional to $\left\langle\psi_{G} \mid \phi_{G}\right\rangle$. Both the harmonic amplifier behavior and the associated transient growth of initial perturbations are linked to the special nonnormality of the linear evolution operator associated with open flows, which may become strong when the flow is nearly parallel because then the supports of direct and adjoint Global modes are well separated in the streamwise direction. For the cylinder wake below threshold, the non-normality is moderate because the maximum of the local scalar product $\left|\psi_{G}(x, y)^{*} \phi_{G}(x, y)\right|$ is 0.04 (Figure $5 c$ ), when the maxima of the local energy $\left|\psi_{G}\right|$ and $\left|\phi_{G}\right|$ are normalized to unity. As a result, the gain in maximum local energy is limited to approximately a factor of 20 .

Figure $6 b$, adapted from Le Gal \& Croquette (2000), demonstrates that the cylinder wake below threshold ( $R e=35$ in the figure) develops a transient von Kármán vortex street when an impulsive displacement of the cylinder is applied. This corresponds to a transient amplification of the perturbation energy. At 
$R e=35$ the wake is globally stable and all direct Global modes are damped. The transient growth illustrated in Figure $6 b$ is therefore the result of non-normality.

3.2.2. SENSITIVITY OF THE GLOBAL SPECTRUM, EFFECT OF FEEDBACK, AND CLOSEDLOOP CONTROL As in the previous toy model (Equation 6), the linear evolution operator is non-normal and small perturbations may modify its spectrum and affect the threshold Reynolds number (see Giannetti \& Luchini 2003 for a more general discussion). Equation 14 is still formally valid and variations of the basic flow will have a larger impact if they occur in the wave maker region where $\left|\psi_{G}(x, y)^{*} \phi_{G}(x, y)\right|$ is large (Figure $5 c$ ). This moon-shaped domain is strikingly similar to the region where the presence of a small control cylinder effectively suppresses vortex shedding, as illustrated in Figure $5 d$, adapted from Strykowski \& Sreenivasan (1990). The magnitude of $\left|\psi_{G}(x, y)^{*} \phi_{G}(x, y)\right|$, which quantifies the receptivity to basic flow modifications, predicts reasonably well the region where the control cylinder is efficient (Figure $5 d$ ). This region is fundamentally distinct from the domain where the flow is most receptive to local forcing, as given by the magnitude of $\left|\psi_{G}\left(x_{f}, y_{f}\right)\right|$.

As remarked in section 3.1.2, the above considerations may explain why it should be sufficient, in numerical simulations, to compute the flow in a box that includes the wave maker region, even if the Global mode is still growing in space at the outlet. This has been observed for the primary bifurcation of the cylinder wake (Giannetti \& Luchini 2003) but also for the secondary bifurcation of the Kármán vortex street (D. Barkley, personal communication).

It is important to note that, for the incompressible Navier-Stokes equations, the pressure is a nonlocal field and it may induce long-distance coupling, which provides a very efficient perturbation to the linear evolution operator, similar to the nonlocal feedback introduced in section 3.1.2. For the cylinder wake, the spectrum modification due to this nonlocal perturbation should be limited because the non-normality is moderate. This is not true for mixing layers and the selfsustained oscillations induced by the addition of a downstream edge, as in edgetone experiments, may be viewed as the destabilization of a Global mode by the resulting nonlocal pressure field. Similarly, numerical simulations of mixing layers often exhibit artificial pressure feedback (Buell \& Huerre 1988), which can destabilize a Global mode. Such modifications of the global spectrum can easily be induced by small nonlocal perturbations because non-normality due to basic flow advection is large.

3.2.3. EFFECT OF NON-NORMALITY ON THE NATURE OF THE BIFURCATION The bifurcation to a Global mode is described by the Landau equation (Equation 9), and the expected associated dynamics close to threshold has been experimentally observed for the cylinder wake (Provansal et al. 1987), the heated jet (Monkewitz et al. 1990), the counterflow mixing layer (Strykowski \& Niccum 1991), etc. The nonlinear modification of the basic flow $\phi_{B}$ forced by $\left|\phi_{G}\right|^{2}$ is maximum downstream close to the location $x_{\max }$ where the direct Global mode is maximum. To 
restabilize the flow, this modification should become important upstream of $x_{\max }$, in the wave maker region where $\left|\psi_{G}(x, y)^{*} \phi_{G}(x, y)\right|$ is large (Figure $5 c$ and section 3.1.2). The more apart $x_{\max }$ and the wave maker region, the faster the mean flow distortion rises beyond threshold. This prediction based on the specific nonnormality of the global linear operator is confirmed by the numerical simulations of Zielinska et al. (1997) and Noack et al. (2003): These authors observe strong nonlinear modifications of the basic flow capable of affecting the length of the recirculation region close to the cylinder. But, as discussed in section 3.1.2, the cylinder wake is only weakly non-normal and even though outside the wave maker region the mean flow distortion is large, the bifurcation is still properly described by the Landau equation. This is no longer the case if the non-normality of the linear global evolution operator increases as it does when the open flow becomes more parallel. In this case, the direct and the adjoint Global modes separate in space, as a consequence the Landau constant vanishes, and the bifurcation steepens with a fast increase in the Global mode amplitude. Close to threshold, the weakly nonlinear theory is then no longer valid and one should resort to a fully nonlinear approach as described in section 4 .

\section{NONLINEAR GLOBAL MODES AND FRONTS IN WEAKLY NONPARALLEL FLOWS}

The dynamics of weakly nonparallel open flows is particularly intriguing: It is well established from Huerre \& Monkewitz (1990) and a series of analyses, numerical simulations and experiments since then (see the foundation section 2.1) that linear Global mode theory properly describes the physical mechanism responsible for the resonance. Yet the above weakly nonlinear analysis indicates that, immediately beyond threshold, large amplitudes are reached, thereby invalidating the linear analysis.

\subsection{Nonlinear Absolute/Convective Instability and Front Velocity}

Strong nonlinearities may be formally taken into account by extending absolute and convective instability concepts (Chomaz 1992) (Figure 7). The basic state of a system is nonlinearly stable if, for all initial perturbations of finite extent and amplitude, the system relaxes to the basic state everywhere in any moving frame. The system is unstable if it is not stable in the above sense. The instability is nonlinearly convective if, for all initial perturbations of finite extent and finite amplitude, the system relaxes to the basic state everywhere in the laboratory frame. It is nonlinearly absolute if, for some initial condition of finite extent and amplitude, the system does not relax to the basic state everywhere in the laboratory frame.

The criterion for nonlinear absolute instability may be simplified further by considering the speed $v_{f}$ of the front that marks the trailing edge of the nonlinear 


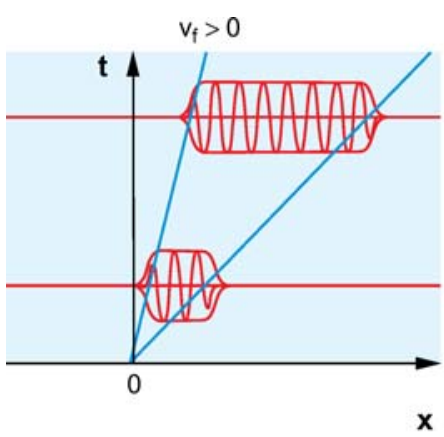

(a)

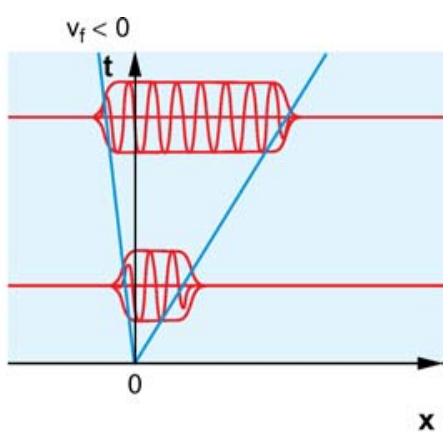

(b)

Figure 7 Diagrams in the $(x, t)$ plane, illustrating the dynamics of a saturated wave packet in an unstable flow, $(a)$ nonlinear convective instability, the velocity $v_{f}$ of the trailing front separating the saturated wave from the basic state is positive, $(b)$ nonlinear absolute instability, the front velocity $v_{f}$ is negative.

wave packet generated by the localized finite amplitude initial perturbation (left front in Figure 7, the basic flow advection being directed to the right by convention). This front separates the basic state upstream (on the left in Figure 7) from the saturated finite-amplitude state downstream (on the right in Figure 7). When its velocity $v_{f}$ is negative (resp. positive) the instability is nonlinearly absolute (resp. nonlinearly convective). The selection problem for the front velocity is well understood in the context of amplitude equations from the studies of Kolmogorov et al. (1937), Dee \& Langer (1983), Dee (1985), Ben-Jacob et al. (1985), van Saarloos (1987, 2003), Powell et al. (1991), van Saarloos \& Hohenberg (1992), etc. For the Ginzburg-Landau equation, Dee \& Langer (1983) observed that the front moves at the speed of the edge of the linear wave packet. This case is presently referred to as a pulled front because the linear region upstream of the front selects the velocity and the oscillation frequency of the entire nonlinear state. Van Saarloos \& Hohenberg (1992), in particular, established that this was not always the case and that, in specific circumstances, the front moves faster upstream than the pulled front. This case is referred to as a pushed front because the saturated wave downstream of the front sets the frequency and the propagation speed of the entire solution (see the review by van Saarloos 2003). The region upstream of the front is then forced by the nonlinear front region and is made of the damped linear wave $k^{-}$that propagates upstream of the front and beats at the front frequency because in the frame of the front the instability is convective (Chomaz \& Couairon 2000).

Direct computations of front velocity in fluid mechanics were recently initiated. Delbende \& Chomaz (1998) numerically computed the linear and nonlinear impulse response of parallel wake flows. The analysis of the linear impulse response along rays $x / t=v$ constitutes a very efficient way to determine the absolute/convective threshold in any Galilean frame, and results from Monkewitz (1988) were thereby recovered without the subtle quest for saddle points of the 
dispersion relation (see Delbende et al. 1998 for details on the method). The nonlinear impulse response was computed with the same numerical code: It was limited by two sharp fronts that coincide with the edges of the linear wave packet. The front separating the von Kármán saturated vortex street from the unperturbed stationary wake, is, therefore, a pulled front: Nonlinearities do not modify its propagation speed. This result is essential and explains, a posteriori, the success of linear absolute instability concepts in predicting the behavior of wakes. Similarly, Cossu et al. (2001) and Chomaz (2004) showed that the front speed is linearly selected (pulled front) for the Blasius boundary layer and for mixing layers, respectively. Even if the front speed has yet to be determined for the majority of classical flows, from now on we mainly consider cases where the selection is linear, i.e., cases where the front is pulled. Linear and nonlinear absolute instabilities coincide in such cases. Front propagation ideas may then be invoked to obtain new fully nonlinear selection principles, as reviewed below.

\subsection{Nonlinear Global Modes in Parallel Flows in a Semi-Infinite Domain}

Rayleigh-Bénard convection with throughflow or Taylor-Couette flow with axial advection are particular open flows because, except in the close vicinity of the inlet, the basic flow is parallel, with viscous diffusion of the throughflow balanced by the pressure gradient. The streamwise inhomogeneity only comes from the inlet where perturbations may be assumed nil. For such semi-infinite parallel flows, if the basic flow is nonlinearly convective, any initial perturbations should eventually vanish because the response it triggers ends up being limited upstream by a front that moves away from the inlet. On the contrary, if the instability is nonlinearly absolute, a nonlinear Global mode should appear because, by definition, the front propagates upstream toward the inlet. This result was confirmed by analyzing several amplitude equations in a semi-infinite domain with the condition of vanishing amplitude at the inlet (Chomaz 1992; Couairon \& Chomaz 1996, 1997ab, 1999b; Tobias et al. 1997, 1998; Worledge et al. 1997). When the front is pulled, a nonlinear Global mode bifurcates when the instability is linearly absolute. At threshold, the selected frequency $\omega_{G}$ is then the absolute frequency $\omega_{0}$, (see section 2.1). The nonlinear Global mode dynamics is governed at leading order by a front blocked at the inlet (Figure $8 b$ ). This front gives rise to a saturated wave beating at the real frequency $\omega_{G}$ and associated with the real wavenumber $k_{N L}\left(\omega_{G}\right)$. Such a saturated wave exists only when $k$ real and $\omega$ real satisfy a nonlinear dispersion relation arising from the solution of a fully nonlinear eigenvalue problem in an infinite and streamwise uniform domain

$$
D_{N L}(k, \omega, R)=0 .
$$

Beyond the global threshold, saturation occurs within a healing length $\Delta_{x}$ (defined, for example, as the distance the perturbation amplitude equals $99 \%$ of the saturation amplitude) from the inlet that decreases as the inverse square root of 


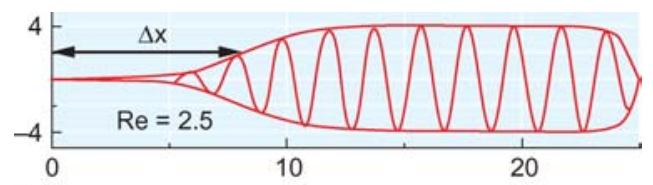

(a)

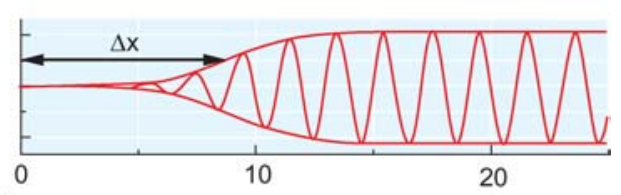

(b)

(c)
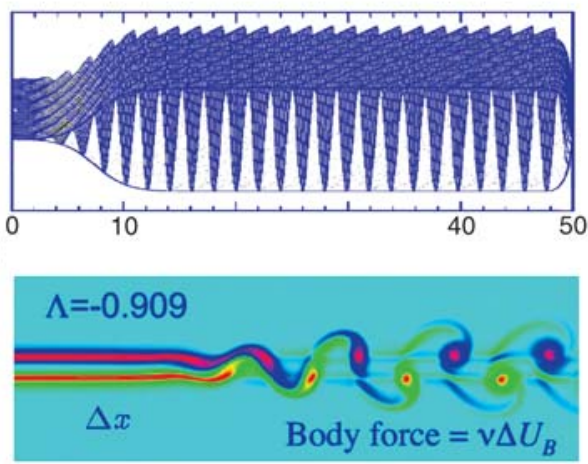

(d)

Figure 8 Nonlinear Global modes in parallel flows, obtained above the absolute instability threshold, by numerical simulation in a semi-infinite domain of: (a) RayleighBénard convection with throughflow (Müller et al. 1992); the vertical velocity in the mid-plane is plotted versus downstream distance and compares very satisfactorily with the Global mode (plotted in $b$ ) obtained by solving the Ginzburg-Landau amplitude equation with complex coefficients for the same parameter values (Couairon \& Chomaz 1997a); (c) Taylor-Couette flow with axial advection (Büchel et al. 1996); the radial velocity in the middle of the gap is presented versus axial distance and versus time as a waterfall; $(d)$ the parallel wake with velocity $U_{0}=0.1$ at center and $U_{\infty}=2.1$ at infinity (corresponding to a velocity ratio $\Lambda \equiv\left(U_{0}-U_{\infty}\right) /\left(U_{0}+U_{\infty}\right)=-0.909$ ) for a Reynolds number $R e=400$ (Chomaz 2003); the vorticity field is presented; the wake is generated directly at the inlet, as in Triantafyllou \& Karniadakis (1990), and it is maintained parallel by the addition of an artificial body force that exactly compensates the viscous diffusion of the basic flow. In all cases, the global frequency at threshold is the absolute frequency $\omega_{0}$ at the inlet and the healing length $\Delta_{x}$ where saturation occurs varies as the inverse square root of departure from criticality, i.e., it decreases as the departure from the absolute instability threshold increases. 
the departure from criticality (Couairon \& Chomaz 1997a). Fineberg \& Steinberg (1987), and Müller et al. $(1989,1992)$ observed this same dynamics in RayleighBénard convection with throughflow (Figure 8a) and Ahlers \& Cannel (1983) and Büchel et al. (1996) observed it in Taylor-Couette flow with axial advection (Figure $8 c$ ). The same dynamics is obtained numerically for the wake instability when the velocity profile is directly imposed at the inlet, as in the study by Triantafyllou \& Karniadakis (1990), and the flow is artificially maintained parallel by a body force that counterbalances the basic flow diffusion (see Figure $8 d$ adapted from Chomaz 2003). A Global mode then appears at the absolute instability threshold predicted by the linear stability analysis of Monkewitz (1988) and its frequency is the absolute frequency at the inlet. The healing length is well fitted by the expression $\Delta_{x} \sim 60.2 *\left(R e-R e_{A}\right)^{-1 / 2}$, where $R e_{A}$ is the critical Reynolds number for absolute instability, a scaling law predicted by the theory.

\subsection{Nonlinear Global Modes in Weakly Nonparallel Flows}

Open flows are usually nonparallel. When the characteristic scale of the downstream variation of the basic flow is comparable to the scale of the instability, nonparallelism is strong and only the fully global theory developed in section 3 applies. When nonparallelism is weak, the global evolution operator becomes highly non-normal, as already explained, and the Global mode amplitude increases extremely fast at threshold. Beyond threshold the nonlinear Global mode should be described by introducing a slow streamwise scale $X=\varepsilon x$ (as in section 2.1.2) and resorting to the front concept for parallel flows (as in section 4.2). Only the dynamics associated with a pulled front will be discussed (see Couairon \& Chomaz 2001 for a discussion when the front is pushed). If a portion of the flow is linearly absolutely unstable, initial perturbations grow until they become nonlinear. They then nucleate a front that starts propagating upstream until its velocity vanishes or until it bumps into the inlet. On the contrary, if the flow is nonlinearly convectively unstable, even perturbations that are large enough to be considered as nonlinear generate a front that moves downstream and is ultimately washed away. Thus nonlinear self-sustained oscillations occur as soon as a finite domain of absolute instability is present. The associated nonlinear Global mode is made up of a front located at the most upstream border of the absolutely unstable domain. The oscillation frequency of the Global mode $\omega_{G}$ is then the absolute frequency at the front location. As in the previous section, the front is followed by a saturated wave beating at the real frequency $\omega_{G}$ associated with the real wavenumber $k_{N L}\left(\omega_{G}, X\right)$ solution of the local nonlinear dispersion relation $D_{N L}\left(\omega_{G}, k_{N L}, X\right)=0$. This border may be a physical boundary of the flow (Figure $3 c$ ), as discussed by Couairon \& Chomaz (1999a, 2001) and Soward (2001) on model equations. Figure $9 c$ displays the Global mode, which emerges from the instability of a nonparallel wake generated at the inlet but free to evolve downstream under the effect of viscosity (Chomaz 2003). The frequency of the wake is then the absolute frequency at the inlet. When the region of absolute instability appears first at the 


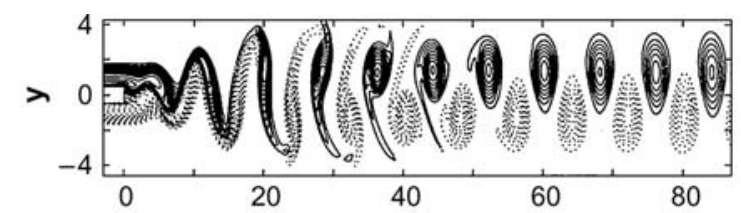

(a)

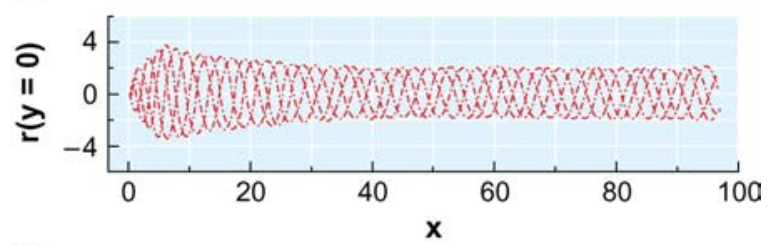

(b)

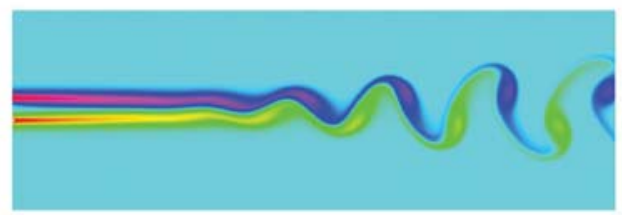

(c)

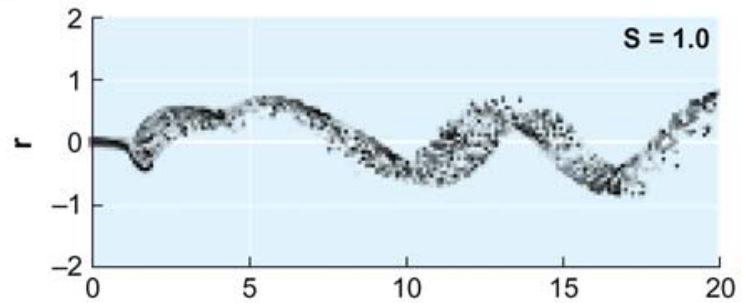

(d)

Figure 9 Nonlinear Global modes obtained in nonparallel flows, above the absolute instability threshold, by numerical simulation of: $(a, b)$ the wake of a blunt-edged plate (Hammond \& Redekopp 1997), (a) vorticity contours, (b) transverse velocity along the centerline versus downstream distance showing a steep front followed by a slowly evolving saturated wave structure; $(c)$ same as Figure $8 d$ but for a nonparallel wake and at $R e=800 ;(d)$ spiral votex breakdown in a swirling jet (Ruith et al. 2003a).

inlet, the Global mode frequency evolves linearly with the departure from criticality, from its leading-order value at threshold $\omega_{c}=\omega_{0}\left(X_{A}=0, R_{c}\right)$. The Global mode steepens at the inlet as the departure from criticality increases (in Figure $9 a b$ notice the steep increase in oscillation amplitude due to vortex shedding in the wake of a blunt-edged plate computed by Hammond \& Redekopp 1997). As a result, the station of maximum Global mode amplitude moves closer to the inlet, the distance to the inlet varying as the healing length, i.e., as the inverse square root of the departure from criticality (Couairon \& Chomaz 1999). These scaling laws and the associated similarity properties were observed experimentally and 


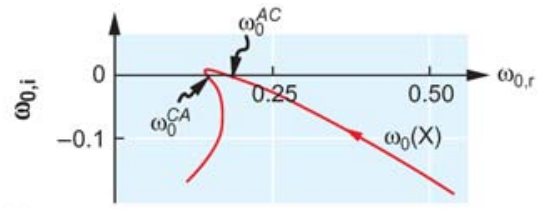

(a)

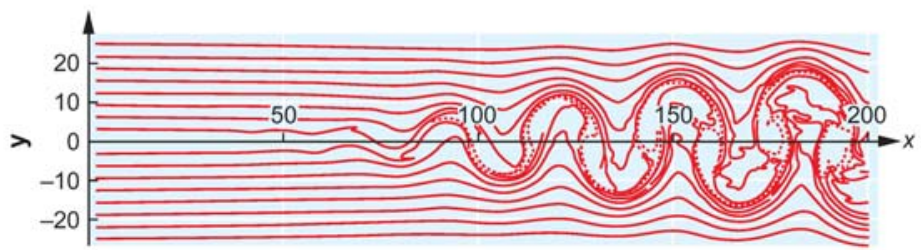

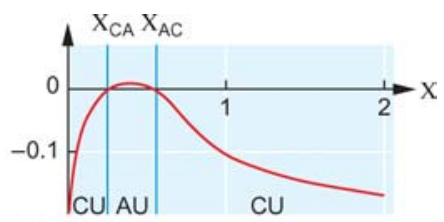

(b)

(c)

Figure 10 Nonlinear Global modes in a synthetic wake at $R e=100$ from Pier \& Huerre (2001); (a) Locus of local absolute frequency $\omega_{0}(X)$ in the complex frequency plane. $(b)$ Local absolute growth rate $\omega_{0 i}(X)$ as a function of streamwise distance. (c) Streaklines corresponding to the self-sustained global structure. Here $\epsilon=1 / \operatorname{Re}=$ 0.01 , and $X=\epsilon x$. The absolute domain is therefore located around $x=50$, where the amplitude of the Global mode starts becoming noticeable but is not yet saturated.

numerically in cylinder wakes with different cross-sections by Goujon-Durand, Jenffer \& Wesfreid (1994), Zielinska \& Wesfreid (1995), and Wesfreid et al. (1996).

The other case, where the absolute region is isolated within the fluid (Figure 3b), was considered by Meunier et al. (1997), Pier et al. (1998), Harris et al. (2000), Pier et al. (2001), and Pier (2002). For a wake profile made absolutely unstable in an isolated region by applying a suitable adverse pressure gradient, Pier \& Huerre (2001) showed that the Global mode shape is similar to the sketch of Figure $3 d$ : A front is located at the upstream border $X_{C A}$ of the absolutely unstable domain (Figure 10). The observed Global mode frequency $\omega_{G}=0.186$ is, as predicted, the absolute frequency prevailing at the station $X_{C A}$ i.e., $\omega_{0}^{C A} \equiv \omega_{0}\left(X_{C A}\right)=0.190$.

Similar results were obtained in a swirling jet (Figure $9 d$ ): As shown by Ruith et al. (2003a), the occurrence of spiral breakdown in swirling jets is due to the global destabilization of the axisymmetric vortex breakdown state. According to the results of Ruith et al. (2003b), this destabilization is linked to the appearance of a large enough domain of absolute instability for the azimuthal mode $m=1$ in the wake of the vortex breakdown bubble. The frequency of the Global mode is the absolute frequency at the upstream border of the absolutely unstable domain $X_{C A}$ and the local amplitude of the mode $m=1$ rises abruptly to order unity at $X_{C A}$. The same shape and frequency selection mechanism holds for the separated bubble in a boundary layer downstream of a double-bump topography (Figure 1) as determined by Marquillie \& Ehrenstein (2003). 


\subsection{Secondary Instability of Nonlinear Global Modes}

In the weakly nonparallel case, the nonlinear Global mode becomes globally unstable when the saturated wave that follows the front becomes absolutely unstable (Brevdo \& Bridges 1996; Chomaz et al. 1990, 1999; Couairon \& Chomaz 1999b; Huerre 1987; Tobias et al. 1998). When the saturated state generated by the primary absolute instability, for example the saturated vortex street, is convectively unstable, the bifurcation scenario is relatively classical. In particular, a single frequency appears at threshold and, if the control parameter is further increased so that the secondary instability becomes, itself, absolutely unstable, a second frequency appears. But, when the saturated wave is absolutely unstable with respect to secondary perturbations at the Global mode threshold, perturbations in the lee of the primary front never settle down and the first bifurcation directly gives rise to complex behavior in the form of a two-frequency mode or a disordered solution at the first instability threshold (Chomaz et al. 1990, Couairon \& Chomaz 1999b, Tobias et al. 1998).

Brancher \& Chomaz (1997) determined the absolute/convective nature of the secondary instability for a single row of finite-size co-rotating vortices as in a mixing layer after saturation of the primary Kelvin-Helmhotz instability. The family of 2D basic flow solutions of the Euler equations discovered by Stuart (1967) describes a $\pi$-periodic array of $2 \mathrm{D}$ vortices separating two counter-flows $U_{ \pm \infty}= \pm 1$. This family of flows is characterized by the nondimensional parameter $\rho \in[0,1]$, which is a measure of the vortex concentration. The solution for $\rho=0$ corresponds to the parallel hyperbolic tangent velocity profile, i.e., infinitely spread out vortices, and the solution for $\rho=1$ consists of a periodic street of point vortices, i.e., infinitely concentrated vortices. The temporal stability of the Stuart vortex street was studied by Pierrehumbert \& Widnall (1982) and more recently by Potylittsin \& Peltier (1999). Brancher \& Chomaz (1997) performed a spatiotemporal stability analysis and found, both for the 2D and 3D instabilities, that the more concentrated the vortices, the less backflow is needed to trigger absolute instability. In particular, for all the concentration parameters tested, the backflow needed to have a secondary absolute instability (2D or 3D) is smaller than the one needed to have a primary absolute instability (Huerre $\&$ Monkewitz 1985). For mixing layers, the secondary pairing and translative instabilities therefore become absolute sooner than the primary Kelvin Helmholtz instability. These results on the pairing instability explain why the 2D Global mode in the parallel mixing layer computed by Chomaz (2004) is irregular at threshold, with pairings occurring randomly. This mechanism may provide an alternative interpretation of the subharmonic resonance observed in forced jets or backward-facing-step flows, which is classically attributed to an acoustic feedback (Broze \& Hussain 1994, Ho \& Huerre 1984, Narayanan \& Hussain 1996). It also predicts that 3D instability may become resonant and therefore extremely intense when the primary instability is forced or is self-sustained. This might account for the occurrence of intense side jets in round jet experiments (Monkewitz et al. 1990), or the 3D instability of separated flows in boundary layers (Kaiktsis et al. 1996, Marquillie \& Ehrenstein 2003). 
This new one-step scenario to disorder, involving a Global mode made of a wave already absolutely unstable to secondary instability at the global threshold, may also explain the abrupt transition to turbulence observed in the rotating disk by Lingwood (1995, 1996, 1997ab). Pier (2003) recently computed the finiteamplitude crossflow vortices that result from the saturation of the absolutely unstable wave in the Ekman layer on a rotating disk and studied its secondary stability properties. The saturated basic flow is computed by direct numerical simulation in a box periodic in both the radial and azimuthal directions, the wavelength in each direction being that predicted by the absolute instability theory of Lingwood (1995). This saturated wave is then considered the new basic flow and it is absolutely unstable. This result contrasts with analogous studies by Koch (2002) and Brandt et al. (2003) on finite-amplitude crossflow vortices in a swept-wing boundary layer and saturated streaks in the Blasius boundary layer, respectively: Both analyses demonstrate that finite-amplitude crossflow vortices and streaks are either stable or convectively unstable to secondary perturbations. Possibly a critical value for the sweep angle exists beyond which the primary vortices become absolutely unstable on wings as they do on the disk, but it has not yet been determined. The other more likely possibility is that the circular disk is specific because perturbations recirculate along the perimeter at any radial location and therefore propagation has only to be considered radially, whereas on a wing the propagation should be considered in a 2D framework (Brevdo 1991) along the span and along the chord. Although the swept-wing and the rotating disk boundary layers are formally equivalent, the conditions on perturbations are not and the primary instability may be absolute only on the disk because of azimuthal periodicity (Lingwood 1997b). The same may be true for the secondary instability and the propagation of perturbations along the chord may explain why they never become absolutely unstable in a swept wing.

Global modes may also lead to disorder when the saturated waves that follow the front disappear downstream through a saddle-node bifurcation as the basic flow evolves (Couairon \& Chomaz 2001).

\section{CONCLUDING REMARKS}

This review brings to the fore the local and global duality of nonparallel flow instabilities which, in a way, are analogous to the particle/wave duality underlying the theory of light [or, more generally, quantum mechanics (Cohen-Tannoudji et al. 1997)].

From the global (particle!) point of view, the specificity of open flows lies in the non-normality of the evolution operator given by the presence of the downstream advection term $U_{B} \partial / \partial x$. This non-normality is specific because it corresponds to a streamwise separation between the adjoint and direct Global modes. All the linear (transient growth, amplifier behavior, closed-loop control, feedback, etc.) and weakly nonlinear (mean flow distortion, Hopf bifurcation) properties of open flows may be understood and measured based on the concepts developed for nonnormal operators. In particular, the degree of non-normality may be measured 
by the scalar product between the adjoint and the direct Global mode, which may constitute an alternative means to discriminate between weakly and strongly nonparallel flows. A first extension stemming from the present article would be to determine this scalar product for all Global modes that are now accessible via direct stability analysis. When non-normality is strong, the global eigenvalue spectrum is no more a robust quantity because small perturbations may stabilize or destabilize the flow even far from threshold. A second proposition would be to compute the pseudospectrum of the operator that would quantify the sensitivity of Global modes to external forcing, noise, basic flow modifications, computer errors, etc. When non-normality is strong, the bifurcation is abrupt and the weakly nonlinear analysis becomes invalid soon beyond threshold.

The local point of view, i.e., the wave interpretation, then becomes more appropriate. In this case, the linear global instability may be linked to the appearance of a region of absolute instability where feedback occurs through the upstream propagation of instability waves. Naturally, this mechanism is only one among others because before the appearance of an absolute instability region, the linear global evolution operator is already strongly non-normal and any small feedback effects may destabilize the flow (pressure feedback as in Schmid \& Henningson 2002, recirculations as invoked by Villermaux \& Hopfinger 1994, coupling involving more than two waves as in Yakubenko 1997). But the linear Global mode structure is not preserved by nonlinearities, which soon after the global threshold become too intense. Fully nonlinear solutions should then be constructed without referring to their linear counterpart by invoking the front concept. When an absolute instability region is present in the flow and when the front velocity is linearly selected (pulled front), the solution is fully nonlinear and corresponds to a front either blocked at the inlet or at rest at the upstream boundary of the absolute instability domain. The front is the wave maker and imposes its frequency to the entire flow, which is thus determined by the absolute frequency at the most upstream border of the absolutely unstable region; it is followed by the saturated solution that beats at this same frequency. Transition to disorder may then occur when the saturated wave becomes absolutely unstable or disappears through a saddle-node bifurcation.

If a pushed (nonlinearly selected) front is selected instead, a fully Global mode may still be constructed but it appears while the flow is still linearly convectively unstable. Its front is then steeper than the imaginary part of the absolute wavenumber would imply and the connection with linear theory is lost. A third proposition would be to compute the front velocity by using, for example, the numerical technique introduced by Delbende \& Chomaz (1998) for all the globally unstable and weakly nonparallel flows. Hopefully, some of them will exhibit a nonlinear front velocity selection (pushed front), for which a strong departure from linear predictions was already demonstrated in the context of model equations. In real flows, a nonlinear absolute instability implying the existence of a pushed front was recently observed in shear layers in a Hele-Shaw cell (Gondret et al. 1999) with new scaling laws for the transition and the existence of hysteresis. But the reason why the majority of open flows seem to follow a linear front velocity selection is still 
mysterious and it should ultimately be interpreted and modeled before concluding that the present theory is complete.

\section{ACKNOWLEDGMENTS}

Patrick Huerre and Larry Redekopp should be warmly thanked for the initial impulse they gave me, their generous collaboration, and their friendship. All the students who taught me so much should also be acknowledged. This project would not have been completed without the friendly support and warm spirit of the LadHyX team, and I thank all its members.

\section{LITERATURE CITED}

Ahlers G, Cannel DS. 1983. Vortex-front propagation in rotating Couette-Taylor flow. Phys. Rev. Lett. 50:1583-86

Aiken CM, Moore AM, Middleton JH. 2003. Non-normal perturbation growth in idealised island and headland wakes. Dyn. Atmos. Oceans 37:171-95

Barkley D, Henderson RD. 1996. Floquet stability analysis of the periodic wake of a circular cylinder. J. Fluid Mech. 322:21541

Bender CM, Orszag SA. 1978. Advanced Mathematical Methods for Scientists and Engineers. New York: McGraw-Hill

Bers A. 1975. Linear waves and instabilities. In Physique des Plasmas, ed. C De Witt, J Peyraud, pp. 117-215. New York: Gordon \& Breach

Brancher P, Chomaz JM. 1997. Absolute and convective secondary instabilities in spatially periodic shear flows. Phys. Rev. Lett. 78:65861

Brandt L, Cossu C, Chomaz JM, Huerre P, Henningson DS. 2003. On the convectively unstable nature of optimal streaks in boundary layers. J. Fluid Mech. 485:221-42

Brevdo L. 1991. 3-Dimensional absolute and convective instabilities, and spatially amplifying waves in parallel shear flows. Z. Angew. Math. Phys. 48:290-309

Brevdo L, Bridges T. 1996. Absolute and convective instabilities of spatially periodic flows. Philos. Trans. R. Soc. London Ser. A 354:1027-64

Broze G, Hussain F. 1994. Nonlinear dynamics of forced transitional jets: periodic and chaotic attractors. J. Fluid Mech. 263:93132

Büchel PB, Lücke M, Roth D, Schmitz R. 1996. Pattern selection in the absolutely unstable régime as a nonlinear eigenvalue problem: Taylor vortices in axial flow. Phys. Rev. E 53:4764-77

Buell JC, Huerre P. 1988. Inflow/outflow boundary conditions and global dynamics of spatial mixing layers. In Studying Turbul. Using Numerical Simulation Databases-II. NASA Ames/Stanford Cent. Turbul. Res. Rep. CTR-S88:19-27

Butler KM, Farrell BF. 1992. 3-dimensional optimal perturbations in viscous shear-flow. Phys. Fluids A 4:1637-50

Chomaz JM. 1992. Absolute and convective instabilities in nonlinear systems. Phys. Rev. Lett. 69:1931-34

Chomaz JM. 2003. Fully nonlinear dynamiques of parallel wakes. J. Fluid Mech. 495:57-75

Chomaz JM. 2004. Transition to turbulence in open flows: what linear and fully nonlinear local and global theories tell us. Eur. J. Mech. B. 23:385-99

Chomaz JM, Couairon A. 1999. Against the wind. Phys. Fluids 11:2977-83

Chomaz JM, Couairon A. 2000. Propagating 
pattern selection and causality reconsidered. Phys. Rev. Lett. 84:1910-13

Chomaz JM, Couairon A, Julien S. 1999. Absolute and convective nature of the Eckhaus and zigzag instability with throughflow. Phys. Fluids 11:3369-73

Chomaz JM, Huerre P, Redekopp LG. 1988. Bifurcations to local and Global modes in spatially-developing flows. Phys. Rev. Lett. 60:25-28

Chomaz JM, Huerre P, Redekopp LG. 1990. Effect of non-linearity and forcing on Global modes. In New Trends in Non-linear Dynamical and Pattern-Forming Phenomena, ed. $\mathrm{P}$ Coullet, P Huerre, pp. 259-74. NATO ASISer. $B$. New York: Plenum

Chomaz JM, Huerre P, Redekopp LG. 1991. A frequency selection criterion in spatiallydeveloping flows. Stud. Appl. Math. 84:11944

Cohen-Tannoudji C, Diu B, Laloe F. 1997. Quantum Mechanics. New York: Wiley

Cossu C, Chomaz JM. 1997. Global measures of local convective instability. Phys. Rev. Lett. 78:4387-90

Cossu C, Chomaz JM, Henningson D. 2001. 2D nonlinear front propagation in the Blasius boundary layer. Bull. Am. Phys. Soc. 46:29

Couairon A, Chomaz JM. 1996. Global instabilities in fully nonlinear systems. Phys. Rev. Lett. 77:4015-18

Couairon A, Chomaz JM. 1997a. Absolute and convective instabilities front velocities and Global modes in nonlinear systems. Physica D 108:236-76

Couairon A, Chomaz JM. 1997b. Pattern selection in the presence of a cross flow. Phys. Rev. Lett. 79:2666-69

Couairon A, Chomaz JM. 1999a. Fully nonlinear Global modes in slowly varying flows. Phys. Fluids 11:3688-703

Couairon A, Chomaz JM. 1999b. Primary and secondary nonlinear global instability. Physica D 132:428-56

Couairon A, Chomaz JM. 2001. Pushed Global modes in weakly inhomogeneous subcritical flows. Physica D 158:129-50

Dee G. 1985. Dynamical properties of propa- gating front solutions of the amplitude equations. Physica D 15:295-304

Dee G, Langer J. 1983. Propagating pattern selection. Phys. Rev. Lett. 50:383-86

Delbende I, Chomaz JM. 1998. Nonlinear convective/absolute instabilities in parallel twodimensional wakes. Phys. Fluids 10:272436

Delbende I, Chomaz JM, Huerre P. 1998. Absolute and convective instabilities in the Batchelor vortex: a numerical study of the linear impulse response. J. Fluid Mech. 355:22954

Drazin PG, Reid WH. 1981. Hydrodynamic Stability. Cambridge, UK: Cambridge Univ. Press

Edwards WS, Tuckerman LS, Friesner RA, Sorensen DC. 1998. Krylov methods for the incompressible Navier-Stokes equations. $J$. Comp. Phys. 110:82-102

Fauve S. 1998. Pattern forming instabilities. In Hydrodynamics and Nonlinear Instabilities, ed. C Goldrèche, P Manneville, pp. 387489. Cambridge, UK: Cambridge Univ. Press See Goldrèche \& Manneville 1998, pp. 387489

Fineberg J, Steinberg V. 1987. Vortex-front propagation in Rayleigh-Bénard convection. Phys. Rev. Lett. 58:1332-35

Giannetti F, Luchini P. 2003. Receptivity of the circular cylinder's first instability. Proc. 5th Eur. Fluid Mech. Conf., Toulouse

Goldrèche C, Manneville P, eds. 1998. Hydrodynamics and Nonlinear Instabilities. Cambridge, UK: Cambridge Univ. Press

Gondret P, Ern P, Meignin L, Rabaud M. 1999. Experimental evidence of a nonlinear transition from convective to absolute instability. Phys. Rev. Lett. 82:11442-45

Goujon-Durand S, Jenffer P, Wesfreid JE. 1994. Downstream evolution of the Bénard-von Kármán instability. Phys. Rev. E 50:30813

Guckenheimer J, Holmes P. 1983. Nonlinear Oscillations, Dynamical Systems and Bifurcations of Vector Fields. New York: SpringerVerlag.

Hammond D, Redekopp L. 1997. Global 
dynamics of symmetric and asymmetric wakes. J. Fluid Mech. 331:231-60

Harris D, Bassom AP, Soward AM. 2000. An inhomogeneous Landau equation with application to spherical Couette flow in the narrow gap limit. Physica D 137:260-76

Hill DC. 1992. A theoretical approach for analyzing the restabilization of wakes. AIAA Pap. 92-0067

Ho CM, Huerre P. 1984. Perturbed free shear layers. Annu. Rev. Fluid Mech. 16:365424

Huerre P. 1987. On the absolute/convective nature of primary and secondary instabilities. In Propagation in Systems Far from Equilibrium, ed. JE Wesfreid, HR Brand, P Manneville, G Albinet, N Boccara, pp. 340-33. Berlin: Springer-Verlag

Huerre P. 2000. Open shear flow instabilities. In Perspectives in Fluid Dynamics, ed. GK Batchelor, HK Moffatt, MG Worster, pp. 159-229. Cambridge, UK: Cambridge Univ. Press

Huerre P, Monkewitz PA. 1985. Absolute and convective instabilities in free shear layers. J. Fluid Mech. 159:151-68

Huerre P, Monkewitz PA. 1990. Local and global instabilities in spatially developing flows. Annu. Rev. Fluid Mech. 22:473537

Huerre P, Rossi M. 1998. Hydrodynamic instabilities in open flows. In Hydrodynamics and nonlinear instabilities, Goldrèche $\mathrm{C}$, Manneville P, ed., pp. 81-294. Cambridge Univ. Press See Goldrèche \& Manneville 1998, pp. 81-294

Hunt RE, Crighton DG. 1991. Instability of flows in spatially developing media. Proc. $R$. Soc. London Ser. A 435:109-28

Jackson CP. 1987. A finite-element study of the onset of vortex shedding in flow past variously shaped bodies. J. Fluid Mech. 182:2345

Kaiktsis L, Karniadakis GE, Orszag SA. 1996. Unsteadiness and convective instabilities in two-dimensional flow over a backwardfacing step. J. Fluid Mech. 321:157-87

Koch W. 1985. Local instability characteristics and frequency determination of self-excited wake flows. J. Sound Vib. 99:53-83

Koch W. 2002. On the spatio-temporal stability of primary and secondary crossflow vortices in a three-dimensional boundary layer. J. Fluid Mech. 456:85-111

Kolmogorov AN, Petrovsky IG, Piskunov NS. 1937. Investigation of a diffusion equation connected to the growth of materials and application to a problem in biology. Bull. Univ. Moscow Ser. Int. Sect. A 1:1-25

Kyle DM, Sreenivasan KR. 1993. The instability and breakdown of a round variabledensity jet. J. Fluid Mech. 249:619-64

Lauga E, Bewley TR. 2002. The decay of stabilizability with Reynolds number in a linear model of spatially developing flows. Proc. $R$. Soc. London Ser. A 459:2077-95

Lauga E, Bewley TR. 2004. Performance of a linear robust control strategy on a nonlinear model of spatially developing flow. J. Fluid Mech. 512:343-74

Le Dizès S, Huerre P, Chomaz JM. 1993. Nonlinear stability analysis of slowly-varying medias: limitations of the weakly nonlinear approach. In Proc. IUTAM Symp. Bluff-Body Wakes, Dyn. Instab., pp. 147-52. Berlin: Springer-Verlag

Le Gal P, Croquette V. 2000. Visualization of the space-time impulse response of the subcritical wake of a cylinder Phys. Rev. E 62:4424-26

Leu TS, Ho CM. 2000. Control of global instability in a non-parallel near wake. J. Fluid Mech. 404:345-78

Lingwood RJ. 1995. Absolute instability of the boundary layer on a rotating disk. J. Fluid Mech. 299:17-33

Lingwood RJ. 1996. An experimental study of absolute instability of the rotating-disk boundary-layer fow. J. Fluid Mech. 314: 373-405

Lingwood RJ. 1997a. Absolute instability of the Ekman layer and related rotating flows. $J$. Fluid Mech. 331:405-28

Lingwood RJ. 1997b. On the impulse response for swept boundary-layer flows. J. Fluid Mech. 344:317-34 
Mamun CK, Tuckerman LS. 1995. Asymmetry and hopf-bifurcation in spherical Couetteflow. Phys. Fluids 7:80-91

Marquillie M, Ehrenstein U. 2003. On the onset of nonlinear oscillations in a separating boundary-layer flow. J. Fluid Mech. 490: $169-88$

Mathis C, Provansal M, Boyer L. 1984. BenardVon kármán instability - an experimentalstudy near the threshold. J. Phys. Lett. 45: 483-91

Maxworthy T. 1999. The flickering candle: transition to a global oscillation in a thermal plume. J. Fluid Mech. 390:297-323

Meunier M, Proctor M, Sokoloff D, Soward A, Tobias S. 1997. Asymptotic properties of a nonlinear $\alpha$-dynamo wave: period, amplitude and latitude dependence. Geophys. Astrophys. Fluid Dyn. 86:249-85

Monkewitz P, Huerre P, Chomaz JM. 1993. Global linear stability analysis of weakly non-parallel shear flows. J. Fluid Mech. 251: $1-20$

Monkewitz PA. 1988. The absolute and convective nature of instability in two-dimensional wakes at low Reynolds numbers. Phys. Fluids 31:999-1006

Monkewitz PA, Bechert DW, Barsikow B, Lehmann B. 1990. Self-excited oscillations and mixing in a heated round jet. J. Fluid Mech. 213:611-39

Moore AM, Perez CL, Zavala-Garay J. 2002. A non-normal view of the wind-driven ocean circulation J. Phys. Oceanogr. 32:26812705

Müller HW, Lücke M, Kamps M. 1989. Convective patterns in horizontal flow. Europhys. Lett. 10:451-54

Müller HW, Lücke M, Kamps M. 1992. Transversal convection patterns in horizontal shear flow. Phys. Rev. A 45:3714-26

Narayanan S, Hussain F. 1996. Measurements of spatiotemporal dynamics in a forced plane mixing layer. J. Fluid Mech. 320:71-115

Nayfeh AH. 1973. Perturbation Methods. New York: Wiley

Noack BR, Afanasiev K, Morzynski M, Tadmor G, Thiele F. 2003. A hierarchy of low- dimensional models for the transient and post-transient cylinder wake. J. Fluid Mech. 497:335-63

Noack BR, Eckelmann H. 1994. A global stability analysis of the steady and periodic cylinder wake. J. Fluid Mech. 270:297-330

Oertel H. 1990. Wakes behind blunt bodies. Annu. Rev. Fluid Mech. 22:539-64

Pier B. 2002. On the frequency selection finiteamplitude vortex shedding in the cylinder wake. J. Fluid Mech. 458:407-17

Pier B. 2003. Finite-amplitude crossflow vortices secondary instability and transition in the rotating disk boundary layer. J. Fluid Mech. 487:315-43

Pier B, Huerre P. 2001. Nonlinear selfsustained structures and fronts in spatially developing wake fows. J. Fluid Mech. 435:14574

Pier B, Huerre P, Chomaz JM. 2001. Bifurcation to fully nonlinear synchronized structures in slowly varying media. Physica D 148:4996

Pier B, Huerre P, Chomaz JM, Couairon A. 1998. Steep nonlinear Global modes in spatially developing media. Phys. Fluids 10: 2433-35

Pierrehumbert RT. 1984. Local and global baroclinic instability of zonally varying flow. $J$. Atmos. Sci. 41:2141-62

Pierrehumbert RT, Widnall SE. 1982. The twoand three-dimensional instabilities of a spatially periodic shear layer. J. Fluid Mech. 114:59-82

Potylitsin PG, Peltier WR. 1999. Threedimensional destabilization of Stuart vortices: the influence of rotation and elliptique. J. Fluid Mech. 387:205-26

Powell JA, Newell AC, Jones CKRT. 1991. Competition between generic and nongeneric fronts in envelope equations. Phys. Rev. A 44:3636-52

Provansal M, Mathis C, Boyer L. 1987. BénardVon kármán instability—-transient and forced régimes. J. Fluid Mech. 182:1-22

Reddy SC, Trefethen LN. 1994. Pseudospectra of the convection-diffusion operator. SIAM J. Appl. Math. 54:1634-49 
Roussopoulos K, Monkewitz PA. 1996. Nonlinear modeling of vortex shedding control in cylinder wakes. Physica D 97:26473

Ruith M, Chen P, Meiburg E, Maxworthy T. 2003a. Three-dimensional vortex breakdown in swirling jets and wakes: direct numerical simulation. J. Fluid Mech. 486:331-78

Ruith M, Meiburg E, Huerre P, Chomaz JM, Gallaire F. 2003b. The role of hydrodynamic instability on the mode and frequency selection of vortex breakdown. Bull. Am. Phys. Soc. $48: 126$

Schmid PJ, Henningson DS. 2001. Stability and Transition in Shear Flows. New York: Springer-Verlag

Schmid PJ, Henningson DS. 2002. On the stability of a falling liquid curtain. J. Fluid Mech. 463:163-71

Soward A. 2001. Thin aspect ratio $\alpha \omega-$ dynamos in galactic discs and stellar shells. In Advances in Nonlinear Dynamos, ed. M Nunez, A Ferriz-Mas. The Fluid Mechanics of Astrophysics and Geophysics. London/ New York: Taylor \& Francis

Soward AM, Jones CA. 1983. The linear stability of the flow in the narrow gap between two concentric rotating spheres. Q. J. Mech. Appl. Math. 36:19-42

Sreenivasan KR, Raghu S, Kyle D. 1989. Absolute instability in variable density round jets. Exp. Fluids 7:309-17

Strykowski PJ, Krothapalli A, Jendoubi S. 1996. The effect of counterflow on the development of compressible shear layers. $J$. Fluid Mech. 308:63-96

Strykowski PJ, Niccum D. 1991. The stability of countercurrent mixing layers in circular jets. J. Fluid Mech. 227:309-43

Strykowski PJ, Sreenivasan KR. 1990. On the formation and suppression of vortex 'shedding' at low Reynolds numbers. J. Fluid Mech. 218:71-107

Stuart JT. 1967. On finite amplitude oscillations in laminar mixing layers. J. Fluid Mech. 29:417-40

Sturrock PA. 1958. Kinematics of growing waves. Phys. Rev. 112:1488-503
Theofilis V. 2003. Advances in global linear instability analysis of non-parallel and three-dimensional flows. Prog. Aerosp. Sci. 39:249-315

Tobias SM, Proctor MRE, Knobloch E. 1997. The role of absolute instability in the solar dynamo. Astron. Astrophys. 318:L55-58

Tobias SM, Proctor MRE, Knobloch E. 1998. Convective and absolute instabilities of fluid flows in finite geometry. Physica D 113:472

Trefethen LN. 1997. Pseudospectra of linear operators. Siam Rev. 39:383-406

Trefethen LN, Trefethen AE, Reddy SC, Driscoll TA. 1993. Hydrodynamic stability without eigenvalues. Science 261:578-84

Triantafyllou G, Karniadakis G. 1990. Computational reducibility of unsteady viscous flows. Phys. Fluids A 2:653-56

Tuckerman LS, Bertagnolio F, Daube O, Le Qur P, Barkley D. 2000. Stokes preconditioning for the inverse Arnoldi method. In Continuation Methods for Fluid Dynamics Notes on Numerical Fluid Mechanics, ed. D Henry, A Bergeon, 74:241-55. Vieweg

van Saarloos W. 1987. Dynamical velocity selection: marginal stability. Phys. Rev. Lett. 58:2571-74

van Saarloos W. 2003. Front propagation into unstable states. Phys. Rep. 386:29-222

van Saarloos W, Hohenberg P. 1992. Fronts pulses sources and sinks in generalized complex Ginzburg-Landau equations. Physica $D$ 56:303-67

Villermaux E, Hopfinger EJ. 1994. Periodically arranged co-flowing jets. J. Fluid Mech. 263:63-92

Woodley B, Peake N. 1997. Global linear stability analysis of thin aerofoil wakes. J. Fluid Mech. 339:239-60

Worledge D, Knobloch E, Tobias SM, Proctor MRE. 1997. Dynamo waves in semi-infinite and finite domains. Proc. R. Soc. London Ser. A 453:119-43

Yakubenko PA. 1997. Global capillary instability of an inclined jet. J. Fluid Mech. 346:181200

Yu M-H, Monkewitz PA. 1993. Oscillations in 
the near field of a heated two-dimensional jet. J. Fluid Mech. 255:323-47

Zebib A. 1987. Stability of viscous-flow past a circular-cylinder. J. Eng. Math. 21:15565

Zielinska BJA, Goujon-Durant S, Dusek J, Wes- freid JE. 1997. Strongly nonlinear effect in unstable wakes. Phys. Rev. Lett. 79:389396

Zielinska BJA, Wesfreid JE. 1995. On the spatial structure of Global modes in wake flow. Phys. Fluids 7:1418-24 